\title{
A Survey on Vital Signs Detection Using Radar Techniques and Processing with FPGA Implementation
}

\author{
Ameen Bin Obadi, Ping Jack Soh, Senior Member IEEE, Omar Aldayel, Member IEEE, Muataz \\ Hameed Al-Doori, Senior Member IEEE, Marco Mercuri, Member IEEE and Dominique Schreurs, \\ Fellow, IEEE
}

\begin{abstract}
This paper presents a survey of the state-of-the-art advances in human vital signs detection using radar sensors, their integration and coexistence with communication systems, and their issues in spectrum sharing. The focus of this survey is to review the detection, monitoring, and tracking of vital signs, specifically the respiration rate and heartbeat rate over the recent five years. It is observed that in line with technological advancements, a multitude of radar types operating in a diverse frequency spectra have been introduced with different hardware implementations, considering various detection scenarios, and applying multiple signal processing algorithms. The aim of these researches varies, from enhancing the detection accuracy, improving the processing speed, reducing the power consumption, simplifying the hardware used, lowering implementation costs, and the combinations of them. Besides that, this review also focuses on literature aimed at increasing the detection accuracy and reducing the processing time using FPGAs, prior to benchmarking them against other processing platforms. Finally, a perspective on the future of human vital signs detection using radar sensors concludes this review.
\end{abstract}

Index Terms-Radars, vital sign detection, respiration, heartbeat rate, FPGAs.

\section{INTRODUCTION}

$\mathrm{H}$ uman health status can be mainly determined from the available vital signals that can be acquired directly from the body, whether invasively or non-invasively. Signals such as blood pressure, heart rate, respiration rate, blood oxygen, motion parameters etc., can provide a precursor to indicate the quality of life for an individual. Among them, signals acquired to indicate the heart rate (HR) and respiration rate (RR) are vital physiological signals indicating the health condition of the person.

Heart rate detection and monitoring can indicate the health status of a person's cardiovascular system. The heart rate changes based on how a person reacts to different situations such as fear, illness, depression etc. Likewise, the instability of respiration rate is an early indicator of physiological variability, whether short- or long term. Thus, HR and RR can be used in various applications, such as sleep monitoring, elderly health home-monitoring, infants or preterm condition monitoring, post-surgery monitoring and trapped victim detection in search and rescue operations [1].
The topic of human vital sign detection has been attracting the interest of many researchers in recent years and has been enabled using different methods and technologies. While some researchers use contact-type methods to detect human vital signs, such as wearable devices and sensors to perform measurements when attached to the human body, other researchers tend to use contactless technologies such as radar systems, cameras and laser technologies. Non-invasive radarbased detection method is preferred by many researchers over other available detection methods. This is because such radar systems reduce the inconvenience caused by wearable devices and electrocardiogram (ECG) equipment. Direct contact methods applied on the human body potentially causes discomfort/harm to the target, and this is especially evident in the case of skin burn injuries, preterm and sleep monitoring. Moreover, the use of direct contact methods may also cause the targets to change their behavior due to awareness and obtrusion caused by the device, thus affecting the measurement accuracy. On the contrary, the application of radar systems eliminates these possibilities and their potential errors.

Besides that, radar-based detection has also been favored in many cases over other non-contact methods due to its applicability for non-line-of-sight monitoring, sensing in foggy environments, and its ability in through-wall detection. Moreover, privacy concern does not arise when using such methods, as no videos or pictures are involved in the detection [1],[2],[3]. In radar-based human vital sign detection, the received signal upon reflection is processed to acquire useful information. Several of the processing steps involved include demodulation, amplification, digitization, transfer, storage, denoising, filtration, and information extraction. These steps are generally categorized as signal acquisition and processing. Radar signals can be processed using different platforms such as Central processing unit (CPU), digital signal processors (DSP), Graphic processing units (GPU), application specific integrated circuits (ASIC)-based processors, field programmable gate arrays (FPGAs), or the combinations of these platforms. Table 1 compares the features of radar-based sensors, ECGs, wearable devices, camera-based sensors, and laser-based sensors for vital sign detection. 
Table 1 Comparison between different technologies for vital sign detection

\begin{tabular}{|c|c|c|c|c|c|}
\hline Feature & Radar & ECG & $\begin{array}{c}\text { Wearable } \\
\text { devices }\end{array}$ & Camera & Laser \\
\hline Convenience & Yes & "No & No & "Yes & "Yes \\
\hline Through wall detection & Yes & N/A & N/A & No & No \\
\hline Privacy concerns & No & No & No & Yes & No \\
\hline Safety concerns & Yes & N/A & N/A & No & No \\
\hline $\begin{array}{l}\text { Dark, foggy } \\
\text { environment }\end{array}$ & Yes & Yes & Yes & No & No \\
\hline $\begin{array}{l}\text { Non-line-of-sight } \\
\text { detection }\end{array}$ & Yes & N/A & N/A & No & No \\
\hline Interference & Yes & No & No & No & No \\
\hline
\end{tabular}

Besides that, it is highly possible that radars and communication devices coexist in the same location. Such coexistence may result in both applications sharing the same spectrum and lead to interference. To facilitate coexistence in the radio spectrum, all radar sensors must comply with regulations of unlicensed operation. The Federal Communication Commission (FCC) in the US allows unlicensed UWB transmission in the 3.1 to $10.6 \mathrm{GHz}$ range with an average transmitted power of less than $-41.3 \mathrm{dBm} / \mathrm{MHz}$ [4]. Radars in the unlicensed frequency band are increasingly being considered for indoor scanning and localization in coexistence with 5G and the Internet of Things (IoT). In other situations, radars and communication devices may utilize the same hardware to reduce cost and complexity. It is also foreseen in the near future that a growing number of communication devices and detection radars coexist and share the spectrum in a heterogenous way. Thus, advancements in techniques to mitigate such coexistence are one of the main issues currently being investigated.

This survey provides a review of the state-of-the-art in this growing research area from the different aspects of processing platforms, detection algorithms, operating frequencies and wireless communication hardware. Such review is the first of its kind, to the best of the authors' knowledge. Some of these researches may not be necessarily applied for vital sign detection but can potentially be used in such application. The rest of this review is organized as follows. The next section will describe and summarize the technical background of radar principles, classifying the types of radar and the processing platforms used, with a specific focus on FPGAs, the signal processing algorithms, the operating frequency spectrum utilized, and the communication of data. Finally, this review ends with a future perspective of potential radar architectures and features that are most suited for applications in vital sign detection. This work intends to highlight the main challenges in vital sign detection using radar techniques and concentrate on its real-time detection aspect to depart from existing reviews available in literature. This is due to the need for alternative solutions and considerations for real-time radar detection, which include innovative parallel processing paradigms on reconfigurable processing devices such as the FPGA.

\section{RADAR BACKGROUND AND PRINCIPLES}

Radars use electromagnetic (EM) waves to detect and monitor remote targets. They transmit and capture reflected radio frequency (RF) waves from one or several targets and process them to obtain information about the targets. In general, a signal source is needed to generate the wave to be transmitted via an antenna. Once the transmitted signal hits the target, a portion of the signal is reflected to the radar while the rest is reflected in other directions or absorbed by the body.

The type and shape of the transmitted signal depends on the radar type. There are four widely used radar types for vital sign detection: continuous wave radar $(\mathrm{CW})$, ultra-wide band impulse radar (UWB-IR), linearly frequency-modulated continuous wave radar (LFMCW) or (FMCW) and step frequency continues wave radar (SFCW). The $\mathrm{CW}$ radar transmits unmodulated continuous wave single tone signals, which can be written as follows [5]:

$$
T(t)=\cos (2 \pi f t+\phi(t))
$$

where $f$ is the oscillation frequency, $t$ is the elapsed time, and $\phi(t)$ is the phase noise caused by the oscillator.

The UWB-IR radars, on the other hand, transmits pulses which are wide in bandwidth. The rate at which these pulses are transmitted per second is called the pulse repetition frequency (PRF). The interval between transmitted pulses is usually used to listen for incoming reflections from objects. The pulse signal is modulated before being amplified and emitted. The transmission signal for the $i t h$ frame, can be written as:

$$
S_{i}(t)=p\left(t-i T_{f}\right) \cos \left(2 \pi f_{0}\left(t-i T_{f}\right)\right)
$$

where $p\left(t-i T_{f}\right)$ is the pulse signal, $t$ is elapsed time, $T_{f}$ is the duration of the frame $\left(T_{f}=\frac{1}{f_{p}}\right), f_{p}$ is the pulse repetition frequency, and $\cos \left(2 \pi f_{0}\left(t-i T_{f}\right)\right.$ is the carrier with the carrier frequency $f_{0}[6]$.

The FMCW radar transmits chirps of sinusoidal signals, which frequency is linearly swept from $f_{0}$ to $f_{1}$. The complex chirp signal can be modeled as follows [7] :

$\mathrm{S}(t)=A_{t} \exp \left(j\left(2 \pi f_{0} t+\pi K t^{2}\right)\right), 0<t<T_{S}$

where $A_{t}$ is the magnitude associated with the power transmitted, $f_{0}$ is the start frequency, $t$ is the time elapsed, and $K$ is the slope of the sweep of frequencies from $f_{0}$ to $f_{1}$ during the duration $T_{S}$. The sweeping bandwidth can then be written as:

$$
B=f_{1}-f_{0}=K T_{s}
$$

The transmitted FMCW waveform is illustrated in Figure 1. 


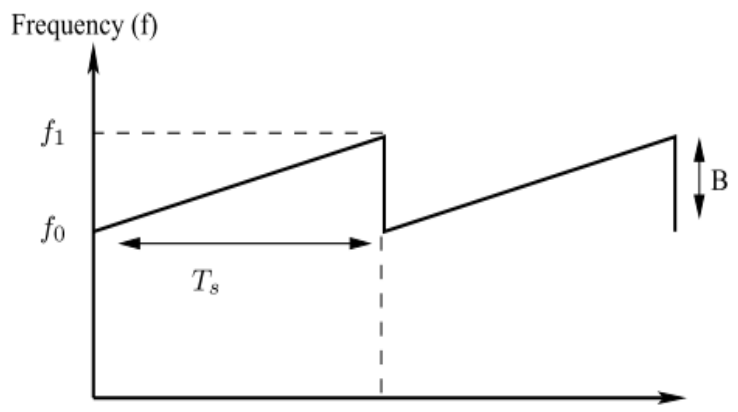

Figure 1: Transmitted FMCW signal with varying frequency in the duration, $T_{S}[8]$

Next is the SFCW radar, which transmits series of discrete tones in a stepwise manner. The waveform of the SFCW consists of $N$ coherent pulses, which frequencies monotonically increases by a fixed increment, $\Delta f$. If the carrier frequency of the first pulse is $f_{0}$, then the $n t h$ pulse frequency is:

$$
f_{n-1}=f_{0}+(n+1) \Delta f
$$

In SFCW radars, the time interval between adjacent pulses is called $\tau$, while the time interval between two groups of $N$ pulses is $N \tau$, with each group is called a burst. The burst time $(N \tau)$ is called the coherent processing interval (CPI). Its concepts are illustrated in Figure 2 [2].

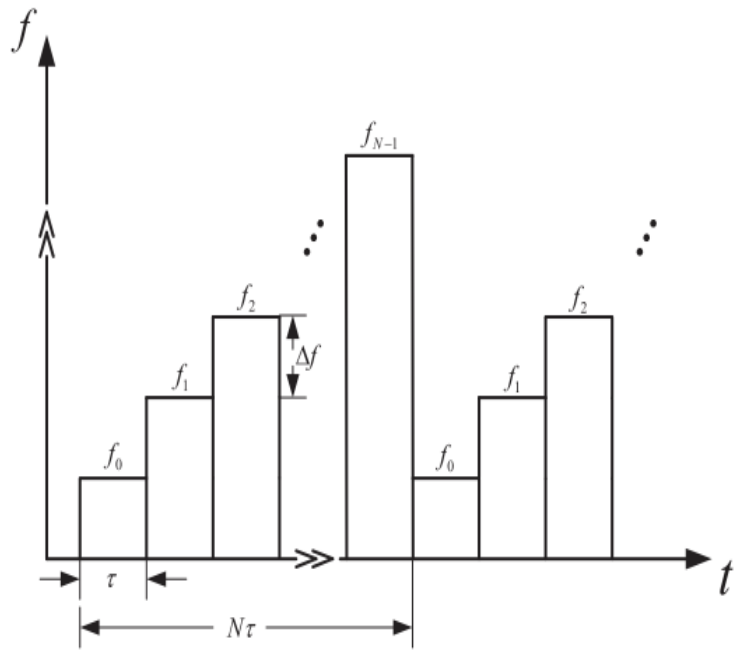

Figure 2 Time frequency representation of SFCW waves [2]

The receiving antenna captures part of the reflected signal, which is then compared with the transmitted signal to extract useful information about the target. Typically, the following four signal parameters are expected to differ between the transmitted and received signal: amplitude, frequency, phase, and polarization. Another major determinant in ensuring radars can extract useful information about the target, is the amount of reflected power captured by the receiver. This factor also determines the maximum radar operating range - the distance below where the radar can correctly detect the target and extract information. The power reflected from the target can be expressed as follows [8]:

$$
P_{\text {ref }}=\frac{P_{t} G_{t} \sigma}{4 \pi R^{2}}
$$

where $P_{t}$ is the transmitted signal power, $P_{r e f}$ is the reflected power, $G_{t}$ is the gain of the transmitting antenna, $\sigma$ is the radar cross section (RCS) of the target, and $R$ is the distance between the radar and the target. It should be noted that the aforementioned equation is a simplified version which assumes no attenuation exist between the radar and the target due to precipitation, cloud or gases. It also assumes that the angular extent of the target is greater than the radar beam width in both azimuth and elevation planes. The received power is:

$$
P_{r}=\frac{P_{t} G_{t} G_{r} \sigma A_{e}}{\left(4 \pi R^{2}\right)^{2}}
$$

where $A_{e}$ is the effective area of receiving antenna and $G_{r}$ is its gain. Based on the previous equation, the maximum radar detectable range, $R_{\max }$ can be calculated as follows [8]:

$$
R_{\text {max }}=\left[\frac{P_{t} G_{t} G_{r} \sigma A_{e}}{16 \pi^{2} S_{\min }}\right]^{\frac{1}{4}}
$$

where $S_{\min }$ is the minimum detectable signal power.

If the reflection is received from a moving target, the wave is modulated by the target motion based on the Doppler effect. The phase of the received signal, $\theta$, can be written as

$$
\theta=\frac{4 \pi d}{\lambda}
$$

where $d$ is the distance to the target and $\lambda$ is the wavelength of the radar signal.

The phase noise is significant in the principle of radar-based detection. It is a characteristic of the signal source and is due to the phase fluctuation within the oscillator. Assuming equation (1) is transmitted, the received signal could be written as [1] :

$R(t)=A_{r} \cos \left(\omega_{0} t+\frac{2 \pi}{\lambda}\left(2 d_{0}+2 x(t)\right)+\phi\left(t-\frac{2 d_{0}}{c}\right)\right)$

where $A_{r}$ is amplitude of received signal, $\omega_{0}$ is the oscillation frequency, $t$ is elapsed time, $\lambda$ is the signal wavelength, $d_{0}$ is the nominal distance between the target and the radar, $x(t)$ is the time varying chest displacement of the target, the term $\phi\left(t-\frac{2 d_{0}}{c}\right)$ is the delayed version of the transmitted phase noise and $c$ is the speed of light. This equation indicates that the phase has been modulated by the chest motion to some extent, and phase demodulation is needed to detect this motion. Moreover, this motion is buried in the phase noise, which may affect the actual phase of the target and, hence, the chest displacement 
accuracy. When using the same source in the transmission and receive, the phase noise of the reflected signal is correlated with the receiver local oscillator. If the time delay between the two signals is small; which is usually the case in vital sign detection, then the phase noise effect can be greatly reduced. This phase noise reduction is usually referred to as range correlation in coherent radar systems. Once the received signal is downconverted by means of multiplication with the transmitted signal and low-pass filtered, the resulting signal can be written as [1]:

$R(t)=1 / 2 A_{r} \cos \left(\frac{2 \pi}{\lambda}\left(2 d_{0}+2 x(t)\right)+\phi\left(t-\frac{2 d_{0}}{c}\right)-\phi(t)\right)$

Since this is a coherent receiver, the phase noise difference is small and can be ignored. The output of the baseband can be written as:

$R(t)=1 / 2 A_{r} \cos \left(\frac{2 \pi}{\lambda}\left(2 d_{0}+2 x(t)\right)\right)$

which presents the relation between chest displacement and the phase of baseband signal [1] .

To account for the Doppler effect in the phase of the received signal, equation (9) is can be modified as follows:

$$
\nabla \theta=\frac{4 \pi \nabla d}{\lambda}
$$

where $\nabla \theta$ is the phase changes caused by the change of position (motion), $\nabla d$. The Doppler modulated frequency of the reflected wave can then be calculated by integrating both sides, resulting in:

$$
f_{d}(t)=\frac{2 v(t)}{\lambda}
$$

where $v(t)$ is the target velocity.

Typically, the received signal containing the Doppler frequency is then channeled through a low noise amplifier (LNA). Next, this signal is down converted into baseband using a mixer and a low pass filter. The remaining signal contains the Doppler frequency, $f_{d}$, caused by the target motion. From this, the target speed can by extracted using equation (14). Another important parameter is $\tau$, which is the total travel time taken by the signal from the transmitter to the receiver after being reflected by the target at a distance $R$ from the transmitter. It can be expressed as follow:

$$
\tau=\frac{2 R}{C}
$$

This parameter is usually associated with the calculation of the range profile in linear frequency modulated continuous wave (LFMCW) radars, or commonly known as FMCW radars. All aforementioned principles and equations are fundamental in the calculation and detection of respiration rate and heart rate [8].

One of the most widely used concepts in literature to model respiration and heartbeat is the "rib cage model". During the respiration, the chest's anterior and lateral diameters expand and shrinks periodically in the anterior-posterior and lateral directions. On the other hand, the heart expands and shrinks periodically in all directions. The changes in the boundaries of the chest wall and the heart in the "rib cage model" are described as sinusoidal oscillations as follows [9]:

$$
\begin{aligned}
& d(t)_{r}=m_{r} \sin \left(2 \pi f_{r} t\right) \\
& d(t)_{h}=m_{h} \sin \left(2 \pi f_{h} t\right)
\end{aligned}
$$

where $d(t)_{r}$ and $d(t)_{h}$ describes the displacement due to respiration and heartbeat, respectively. Parameters $m_{r}$ and $m_{h}$ represent the amplitude of the displacement due to respiration and heartbeat, respectively, $f_{r}$ is the respiratory rate, and $f_{h}$ is the heart rate [9], [10]. Radars can detect these tiny displacements in the human chest due to respiration and heartbeat, as described in by (16) and (17). These displacements modulate the phase of the signals transmitted by the radar, based on the Doppler principle. Thus, the target respiration and heart rate information are embedded in the modulated phase of the received radar signal. The extraction of the vital sign information from the phase of the radar signal can then be performed using a variety of algorithms.

\section{RADAR TYPES FOR VITAL Sign DETECTION}

Generally, vital sign detection is made more effective by selecting the right type of radar. Besides that, practical aspects such as technical requirements and environment in which the vital sign detection takes place determines the suitability of the radar type. These requirements may include typical detection distance, multiple targets detection, moving targets sensing, and through-wall detection. The four potential radar types, namely continuous wave radar $(\mathrm{CW})$, linearly frequency-modulated continuous wave radar (LFMCW) or (FMCW), ultra-wide band impulse radar (UWB-IR) and step frequency continues wave radar (SFCW) and their features, advantages and drawbacks are summarized in Table 2. CW radar is used in vital sign detection for its cost efficiency and design simplicity. However, CW radars do not have range detection capability, they receive reflections from everywhere. As a result, compared to other types of radars, they are less immune to jammers and unwanted reflections. Consequently, their detection is limited for used within short distances. Moreover, a CW radar is not suitable in detecting vital signs of multiple human targets and/or human target localization and tracking. On the other hand, the detection distance in radars is dependent on the type of application. In the case that a long-range detection is needed in the target application, signals with high power and very directive antennas can be introduced. Despite that, for the indoor vital sign detection, the transmit power is limited to $0 \mathrm{dBm} / 50 \mathrm{MHz}$, which then also restricts the maximum detection distance. A FMCW, a UWB impulse, or a SFCW radars can be used in vital sign detection for multiple targets. However, UWB impulse radars are more complex and costlier compared to the $\mathrm{CW}$ radars. On the other hand, both FMCW and SFCW radars are capable of vital sign detection for multiple subjects, localization and tracking, features high SNR, are less complex and more 
cost efficient compared to UWB impulse radars. The preference of SFCW over FMCW signal types in vital sign detection tends to be influenced by factors such as availability of stepped frequency in the radar's transmit signals. Due to this feature, compressive sensing can be applied to this radar type, resulting in improved detection speed [2]. Since $\mathrm{CW}$ radars are less immune to jammers, they typically achieve less SNR compared to the other radar types. Depending on the coherence integration and design, UWB-IR, FMCW and SFCW enable higher process gains and consequently improve SNR relative to $\mathrm{CW}$ radars. The sign ( $\mathrm{x}$ ) in Table 2 indicate that the radar does not possess the specified feature, or it exists with significant limitations.

Table 2 Features of different potential radar types for use in vital sign detection

\begin{tabular}{|l|c|c|c|c||}
\hline \multicolumn{1}{|c|}{ Feature } & CW & FMCW & UWB-IR & SFCW \\
\hline Long distance detection & $x$ & $\checkmark$ & $\checkmark$ & $\checkmark$ \\
\hline High detection accuracy & $\checkmark$ & $\checkmark \checkmark$ & $\checkmark \checkmark$ & $\checkmark \checkmark$ \\
\hline Target speed detection & $\checkmark$ & $\checkmark$ & $\checkmark$ & $\checkmark$ \\
\hline Multiple subjects' detection & X & $\checkmark$ & $\checkmark$ & $\checkmark$ \\
\hline Through wall detection & $\checkmark$ & $\checkmark \checkmark$ & $\checkmark \checkmark$ & $\checkmark \checkmark$ \\
\hline Localization & X & $\checkmark$ & $\checkmark$ & $\checkmark$ \\
\hline Simplicity & $\checkmark \checkmark \checkmark$ & $\checkmark \checkmark$ & $\checkmark$ & $\checkmark \checkmark$ \\
\hline Low cost & $\sqrt{ }$ & $\checkmark \checkmark$ & $\checkmark$ & $\checkmark \checkmark$ \\
\hline Target range & $\checkmark$ & $\checkmark$ & $\checkmark$ & $\checkmark$ \\
\hline Tiny motion detection & $\checkmark$ & $\checkmark \checkmark$ & $\checkmark \checkmark$ & $\checkmark \checkmark$ \\
\hline High SNR & $\checkmark$ & $\checkmark$ & $\checkmark$ \\
\hline
\end{tabular}

The detection of vital signs of a human using radar systems involves the use of electromagnetic frequency spectrum, which also varies depending on the type of radar and their application. One of the main frequency bands used is the industrial, scientific and medical (ISM), a band of radio and microwave frequencies reserved and designated for industrial, scientific and medical equipment that use RF. Besides that, UWB radars are increasing being chosen due to the regulation by the Federal Communication Commission (FCC). This regulation allows unlicensed wireless operation of radars in the UWB band of 3.1 to $10.6 \mathrm{GHz}$ [11][12], [13], with not more than $-41.3 \mathrm{~dB} / \mathrm{MHz}$ of average power transmission. On the other hand, the European Telecommunication Standards Institute (ETSI) also permits the unlicensed operation of communication technologies in the spectrum between 6 and $8.5 \mathrm{GHz}$, whereas the Korean Communication Commission (KCC) permits operation in the frequency band of 7.2 to $10.2 \mathrm{GHz}$ [4],[14], [15].

Besides the radar type and the operating frequency band, several other important considerations in designing radar-based vital sign detection systems include the type of vital signal to be detected and the power consumption level. A comparison of the power consumption levels of several recently reported radars operating between 2 and $15 \mathrm{GHz}$ is presented in

Table 3 [16]. Notice that the highest DC peak power consumption of $148 \mathrm{~mW}$ is observed in [17], whereas the lowest of $19 \mathrm{~mW}$ is presented in [16]. During active detection, the design in [18] consumed the highest DC power of $695 \mathrm{~mW}$, while [16] again featured a remarkably low DC power consumption of $0.68 \mathrm{~mW}$. Such low power consumption levels will enable the effective implementation of battery-powered radar-based sensors. The state-of-the-art literature for radarbased vital sign detection is summarized based on the type of radar in Table 4. The following subsections will present more details of the different radar types and their respective state-ofthe-art literature.

Table 3 Comparison of power consumption of several state-of-the-art radars

\begin{tabular}{|c|c|c|c|c|c|}
\hline & [16] & [4] & [18] & [19] & [17] \\
\hline Freq $(\mathrm{GHz})$ & $6.8-8.2$ & $7.2-8.5$ & $2-5$ & 10 & 15 \\
\hline VDD (V) & $1 / 1.1$ & 1.8 & 1.9 & 1.2 & 1.2 \\
\hline $\begin{array}{l}\text { Chip } \\
\text { Content }\end{array}$ & $\begin{array}{l}\text { ADC, LPF, } \\
\mathrm{HPF}, \mathrm{PA}, \\
\text { DCO, } \\
\mathrm{LNA} / \text { mixer }\end{array}$ & $\begin{array}{l}\text { OSC, PLLs, } \\
\text { PMU, DAC } \\
\text { preamp, } \\
\text { LNA } \\
\text { comparators, } \\
\text { HPF, digital } \\
\text { backend and } \\
\text { control logic }\end{array}$ & $\begin{array}{l}\text { Switch, } \\
\text { LNA, buffer, } \\
\text { S\&H circ, } \\
\text { T\&H, } \\
\text { integrator, } \\
\text { VGA, pulse } \\
\text { gener. timing } \\
\text { circu, power } \\
\text { splitter, } \\
\text { output buff. }\end{array}$ & $\begin{array}{l}\text { 4ch RX } \\
\text { RFFEs, } 4 \\
\text { ch RXBBs, } \\
\text { SPI, DLL } \\
\text { MPS, } \\
\text { DDSs, } \\
\text { PLLs, PSs, } \\
\text { PAs } \\
\end{array}$ & $\begin{array}{l}\text { ADC, } \\
\text { VGA, BPF } \\
\text { Rx RFFE, } \\
\text { DA-PA, } \\
\text { chirp } \\
\text { synthesizer } \\
\text { and DSP } \\
\text { reservation }\end{array}$ \\
\hline $\begin{array}{l}\text { Peak DC } \\
\text { power }\end{array}$ & $19 \mathrm{~mW}$ & N/A & N/A & $141 \mathrm{~mW} / \mathrm{ch}$ & $148 \mathrm{~mW}$ \\
\hline $\begin{array}{l}\text { DC power } \\
\text { during } \\
\text { active }\end{array}$ & $0.68 \mathrm{~mW}$ & $118 \mathrm{~mW}$ & $695 \mathrm{~mW}$ & $141 \mathrm{~mW} / \mathrm{ch}$ & $148 \mathrm{~mW}$ \\
\hline
\end{tabular}

Table abbreviations:

ADC: analogue to digital converter, LPF: low pass filter, HPF: high pass filter, LNA: low noise amplifier, DCO: digitally controlled oscillator, PA: power amplifier, OSC: oscillator, PLL: phased locked loop, PMU: power management unit, DAC: digital to analog converter, T \& H: track and hold amplifiers, S \& $\mathrm{H}$ : sample and hold block, VGA: variable gain amplifier, 4ch RX RFFE: 4 channels receiver RF front end block, 4ch RXBBs: 4channels receiver baseband block, SPI: serial peripheral interphase, DLL MPS: delay locked loop based on multiphase synthesizer, DDS: direct digital synthesizer, PS: phase shifter, BPF: bandpass filter, DA-PA: driver amplifier-power amplifier, DSP: digital signal processing.

Table 4 Radar types for vital sign detection

\begin{tabular}{||c|c|}
\hline Radar Type & \multicolumn{1}{|c|}{ Reference } \\
\hline \hline CW & {$[1],[3],[8],[20],[21],[22],[23],[24],[25],[26]$,} \\
& {$[27],[28],[29],[30],[31],[32],[33],[34],[35]$} \\
\hline UWB-IR & {$[2],[10],[21],[36],[37],[38],[39],[4],[40],[41]$,} \\
& {$[42],[43],[44],[45],[46],[47],[48],[49],[50]$} \\
\hline FMCW & {$[8],[7],[51],[52],[53],[54]$,} \\
\hline SFCW & {$[2],[9],[55],[56],[57][58]$} \\
\hline
\end{tabular}

\section{A. CW Radar}

The authors in [1] used a CW Doppler and a pulse radar to 
wirelessly detect heart signal and breathing signal. Several sources of signal distortion were also introduced into these signals to be evaluated. One of the distortion sources studied in this work is channel imbalance in quadrature receivers. This work also proposed an innovative hardware design using packet radar and low pulse IF receiver architecture to overcome these issues. Next, the work in [3] developed a CMOS direct conversion $\mathrm{CW}$ radar. This radar sensor contains a voltagecontrolled oscillator to generate the $\mathrm{CW}$ signal, and other necessary components such as frequency divider, power amplifier and quasi circulator (QC). This design also includes a clutter canceller block consisting of variable gain amplifier and $360^{\circ}$ phase shifter. This clutter canceller performs cancellation for the transmitted leakage power leakage from the QC and the stationary background reflection clutter. Experimental assessments for the detection of human vital signs at $75 \mathrm{~cm}$ was demonstrated, with the heartbeat and respiration signals clearly observed. Meanwhile, the thesis in [8] introduces a unique architecture based on multidimensional signal processing for directional sensing and remote localization. This architecture integrates CW Doppler radar with array signal processing based on 2-D Infinite Impulse Response (IIR) Spatial Band Pass (SBP) digital filters. The proposed architecture consists of a wideband omnidirectional Uniform Linear Array (ULA) consisting of 64 receiver antennas. It spatially samples the reflected RF waves with each antenna having its own RF front end. Each front end consists of an LNA, band pass filter (BPF), gain amplifier (GA), and phase shifter (PS) to generate the inphase and quadrature components. These signals are then down converted to baseband using mixers which multiply the output of the PS with a copy of the transmitted signal. This copy is generated using a voltage-controlled oscillator (VCO) and 65way splitter. This step is followed by baseband amplification and low pass filtering. The baseband signals are then down sampled using dedicated down-sampling ADCs at each antenna. After that, the signals are digitally processed using 2D IIR SBP beamformer to provide information of the surrounding targets. Although the main application of this work is in micro unmanned aerial system (UAS) detection and automated cyber physical system (CPS), the radar signal extraction methods can be applied to vital sign signal extraction.

Next, in [20], a CW radar and its corresponding processing techniques are used to detect cardiopulmonary activities of human body. Detection is performed at several body positions and scenarios. Meanwhile, a human sensing application radar is presented in [21] using a CW Doppler radar. The CW radar generates a $3 \mathrm{GHz} \mathrm{CW}$ signal which is then amplified through a power amplifier before being transmitted via a Vivaldi antenna. In the receiver side, the received signal is amplified by an LNA. The CW radar utilizes a super-heterodyne receiver to eliminate out-of-band noise and nonlinear distortions by converting the received signal into intermediate frequency (IF) signals, followed by the application of a BPF. Next, the IF signal is digitized, and the sampled data is sent to the FPGAbased digital downconverter. The structure of the $\mathrm{CW}$ radar is shown in Figure 3. Note that the architecture of the super- heterodyne receiver is more complex compared to the radar architecture shown in Figure 5, which enables direct mixing of the received signal with the local oscillator signal. Despite this, the CW radar in Figure 3 enables the elimination of low frequency noise and interference, at the cost of added complexity.

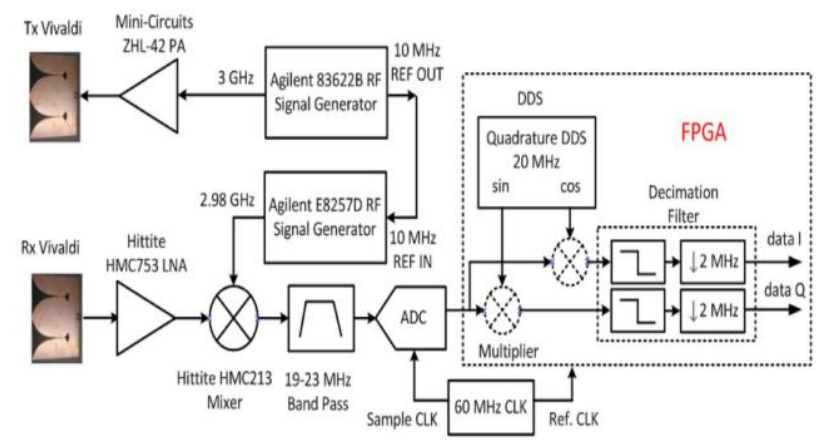

Figure 3 Block diagram of the CW radar prototype [21] @ 2013 IEEE

A recent study [22] demonstrated that a machine learningbased algorithm can be used to model heart beat signal using respiration artifacts. For this purpose, a CW radar is used to measure the results, which confirms that the heartbeat signal is independent and separable from respiration signal. Another interesting study in [23] proposed a time domain detection method using a CW Doppler radar to reduce the time required to obtain a high resolution spectrum of RR and HR. This process typically takes around 30 seconds. The proposed time domain peak detection algorithm is capable in acquiring RR and HR in a breathing cycle (of around 5 seconds). Meanwhile, the design of an SDR prototype for vital sign sensing and detection based on CW radar is presented in [25]. This research is aimed at investigating the validity of remotely sensed of RR and HR. Besides this, another interesting detection method based on CW radar is presented in [26]. Heart rate variability was able to be monitored in real time. The proposed method uses a combined frequency- and time domain technique to obtain the beat-tobeat interval. The low computational complexity of the proposed algorithm makes its application in real time possible. Next, in [27], a CW radar system was developed and implemented on a printed circuit board (PCB) to conduct vital sign detection measurement. It applies a $24 \mathrm{GHz}$ transceiver chip (BGT24MTR11 from Infineon) with homodyne architecture. The prototyped three-layered PCB is shown in Figure 4.

A proposed heart beat model and heart rate variability detection method was presented in [28] based on a $2.4 \mathrm{GHz}$ bistatic quadrature CW Doppler radar. The heart signal was modeled based on Gaussian pulses rather than standard sinusoidal signals. The system hardware generates a single tone continuous wave and transmits it through the antenna. The reflected signal from the target is captured by the receiver antenna, demodulated, amplified and then sampled by the ADC. The signal is then fed into a laptop for processing. Theoretical and experimental studies conducted validated the operation of the radar at $1.5 \mathrm{~m}$ from a human body. Most importantly, the 
proposed method was able to reconstruct the heart-beat signal and determine the heart rate variability accurately. Next, in [29], a $24 \mathrm{GHz}$ vital sign CW Doppler radar was proposed. This radar system adopts a commercially available and cost-effective $24 \mathrm{GHz}$ transceiver module. The received signal is down converted to obtain the I/Q signals, which are then filtered using 4 taps Butterworth bandpass filter with a $30 \mathrm{~Hz}$ cut off frequency. The output of the filtered signal is then amplified 100 times before being digitized using a microcontroller unit. The interesting aspect in this design is its use of a Wireless Local Area Network (WLAN) module to transmit the digitized signal to a PC for further extraction of cardiopulmonary rate. All modules in this work were implemented on a custom PCB to ensure size compactness. Next, a CW radar system to remotely sense human signals outdoors was presented in [30]. It is designed to find survivors after any chemical or nuclear catastrophe. This portable radar which is integrated with advanced respiration signal detection methods is also capable of minimizing interference from other moving objects in the environment. This method was experimentally validated outdoors for a target located at $6 \mathrm{~m}$ distance. The block diagram of the system is shown in Figure 5.

The work in [31] addresses the vital signs and human walking modeling issue. It proposed a new electromagnetic model using a CW radar based on a software defined radio. Meanwhile, researchers in [32] presented a singular spectrum analysis (SSA) method to detect human signals. The reflected signals transmitted by a $\mathrm{CW}$ radar are then processed using SSA. Experiments were conducted on a person located at $2 \mathrm{~m}$ distance behind a wall. The simulation and experimental data validated the effectiveness and accuracy of the proposed SSA method when benchmarked against the use of band pass filters (BPF). Next, a novel architecture for tracking phase modulation caused by cardiopulmonary activities was proposed in [33]. It is based on a CW radar operating as a PLL in demodulation configuration. Its block diagram is shown in Figure 6 and Figure 7. As an alternative, optimization algorithms can be used in place of direct spectral analysis to detect vital signs in different scenarios using a CW Doppler radar [34]. Besides that, the SNR of the reflected signals from human subjects can be improved for a CW radar, as demonstrated in [35]. Classification algorithms were also used to identify six different human subjects in this work, and therefore can be used to detect vital sign fingerprints. Frequencies generally used in $\mathrm{CW}$ radars for detection purposes starts from as low as $2 \mathrm{GHz}$ [32] up to $110 \mathrm{GHz}$ [25], whereas other commonly used bands are centered at $2.4 \mathrm{GHz}, 5.8 \mathrm{GHz}, 10 \mathrm{GHz}, 24 \mathrm{GHz}$, and $60 \mathrm{GHz}$, as summarized in Table 5. Higher sensitivity to chest displacements is exhibited in $\mathrm{CW}$ radars with increasing frequency (towards millimeter-waves) due to the shorter wavelength. On the other hand, millimeter-waves frequency operation attenuates more easily, especially when the signal is transmitted through highly lossy human tissues such as muscles or skin [59].

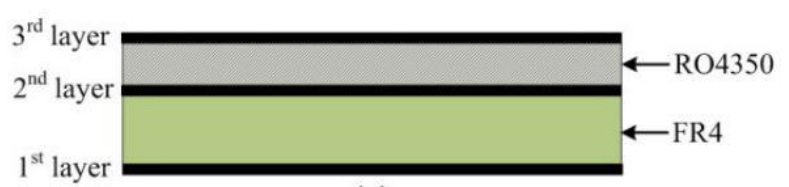

(a)

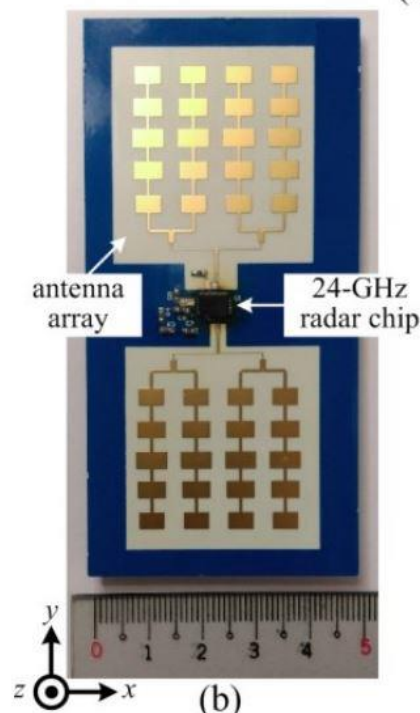

(b)

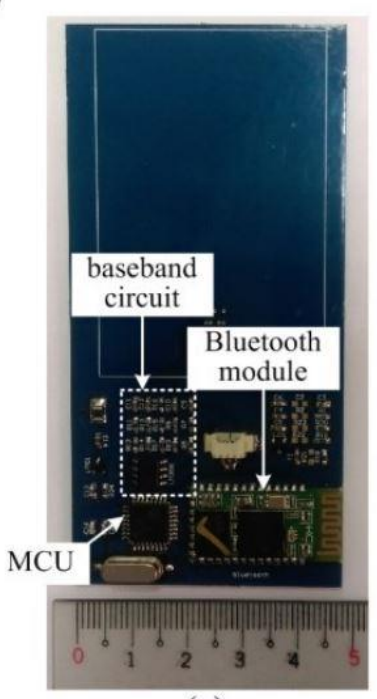

(c)
Figure 4 Photograph of prototyped radar (a) the structure of threelayered PCB (b) top view (c) bottom view [27] @ 2016 IEICE

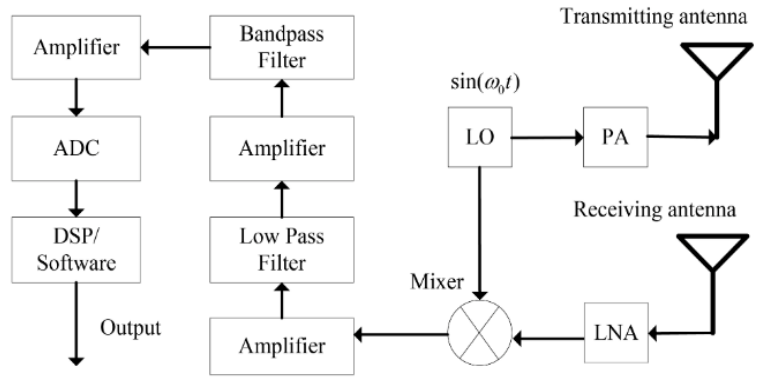

Figure 5 Block diagram of proposed system in [30]. Licensed under Creative Commons attribution license https://creativecommons.org/licenses/by/4.0/

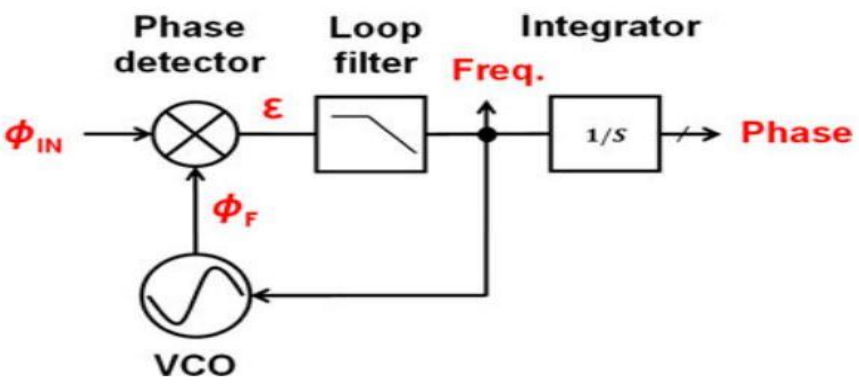

Figure 6 PLL configuration [33] (C) 2018 IEEE 


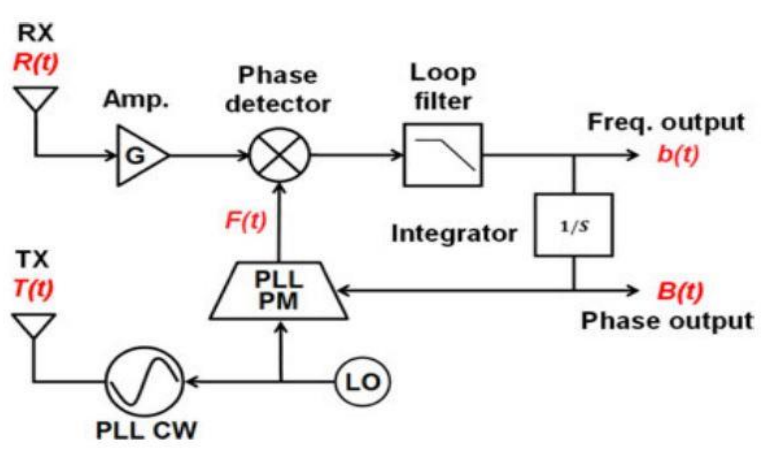

Figure 7 Phase detection [33] @ 2018 IEEE

\section{B. UWB Impulse Radar}

UWB impulse radar operates throughout a wide bandwidth, and consistently transmits narrow pulses (or impulses) to the target. Then, information about the target range can be extracted with high resolution by processing the received echoes. It is also capable of target localization, tiny motion detection and through wall detection. On the other hand, due to the moderately high noise accompanying the signal, UWB-IR typically has low SNR. Moreover, it is more complex in architecture, and thus more costly to implement compared to $\mathrm{CW}$ radars [2],[8].

The application of such radar type in health monitoring includes a novel method proposed to simultaneously extract heart rate and breathing information from echo signals [10]. Another UWB impulse radar used for human sensing application is presented in [21]. This UWB radar utilizes the same carrier frequency of $3 \mathrm{GHz}$ used in the $\mathrm{CW}$ radar described in the previous subsection. In fact, the $\mathrm{CW}$ and the UWB radars of this work shared fundamental elements such as the transmitter and receiver antennas, the power amplifier, the LNA and the mixer for cost-efficiency. In this UWB radar, a 700 ps Gaussian pulse is modulated with a $3 \mathrm{GHz}$ carrier using a mixer and channeled through a high gain power amplifier before being transmitted via a wideband Vivaldi antenna. On the receiver side, an eight-element array collects the signal, with one channel selected at a time using a switch. Next, the signal is passed through an LNA to be down-converted into baseband. The output of the coherent down-conversion is then filtered before being channeled into an amplifier. The output is then sent for digitization based on the equivalent time sampling strategy. The structure of this UWB radar is shown Figure 8.

A more challenging work involving the detection of heartbeats originating from multiple stationary targets at equal distances from an UWB radar is presented by [37]. An algorithm to separate the fundamental frequency of the heartbeat from its harmonics was proposed and analyzed numerically, prior to its experimental validation using two targets. Results from this experiment were then compared with camera-based results. Next, an algorithm was proposed to extract heart rate from respiration signal in [38] using an UWB impulse radar. Different experimental scenarios were performed to prove the validity of this algorithm. Besides that, an auto-correlation method was explored to detect random body movements during experiments. The algorithm was applied to the echoes reflected from stationary and non-stationary targets.
Meanwhile, the effects of speech and hand movements on the measurement of respiration signal using an UWB radar was studied in [39]. It can be observed that the noise from these activities affected the accuracy of the target signal.

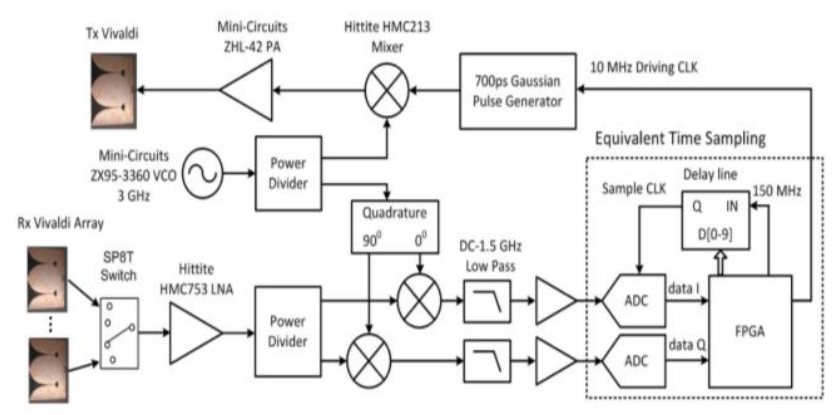

Figure 8 Block diagram of the UWB radar prototype [21] (C) 2013 IEEE

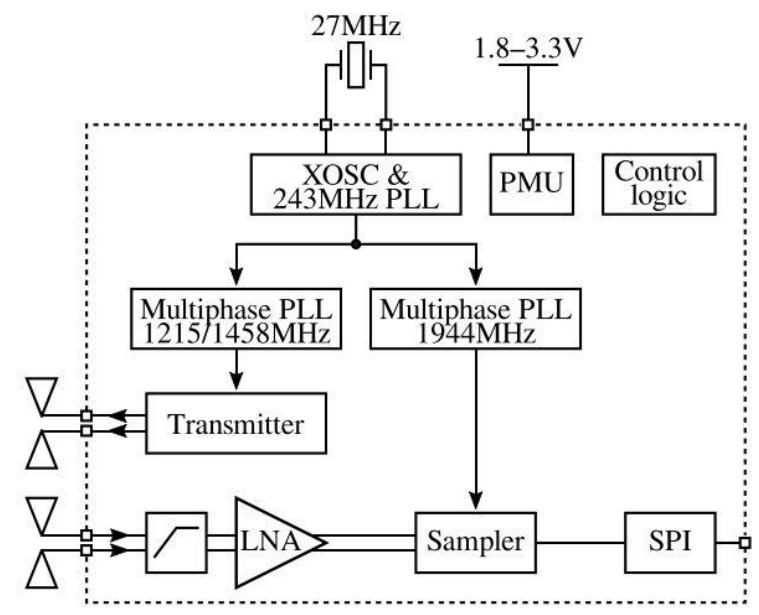

Figure 9 Block diagram of SoC radar in [4]. licensed under Creative Commons attribution license

In terms of hardware development, a $55 \mathrm{~nm}$ CMOS SoCbased pulsed radar was developed in [4] for vital sign detection. The block diagram of the SoC radar system is shown in Figure 9. Successful detection of the radar signals at $5 \mathrm{~m}$ and $9 \mathrm{~m}$ distances were also reported in this work. In [40], a universal software radio peripheral platform (USRP-2954R) was used to implement the impulse radar system illustrated in Figure 10. This system aims to detect displacement and vibration accurately in real time. The time domain cross correlation ranging was performed using an FPGA. Meanwhile, the work in [44] focuses on the signal processing of a low power wireless CMOS impulse radar sensing system. A new reconstruction methodology of the compressive sensing algorithm was proposed. It was implemented on an FPGA and can support real-time human detection. Next, in [45], a signal processing platform for UWB radar used for analyzing human breathing was presented. This platform analyzes new features of human breathing, which have not been investigated using radar 
systems. Examples of these features are inspiration and expiration speeds, respiration intensity and holding ratio. To do so, a new respiration signal model known as the four segments linear waveform (FSLW) respiration model was proposed, with early termination techniques. The radar transceiver diagram is shown in Figure 11.

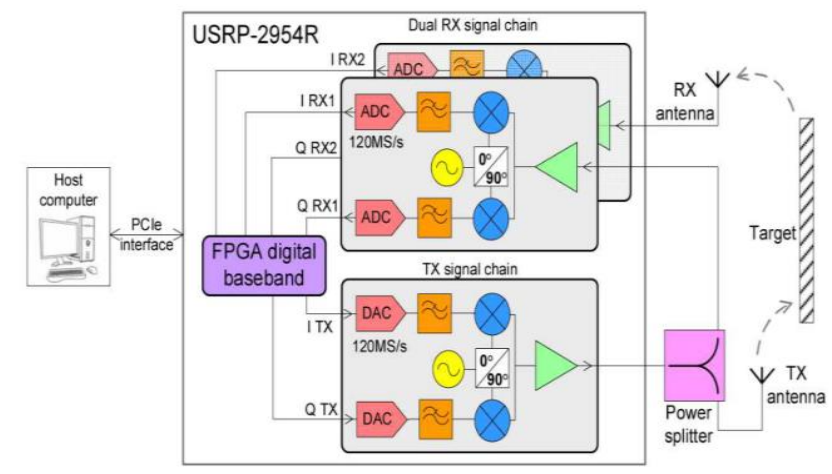

Figure 10 Pulse radar system in [40]. Licensed under Creative Commons attribution licenses https://creativecommons.org/licenses/by/4.0/

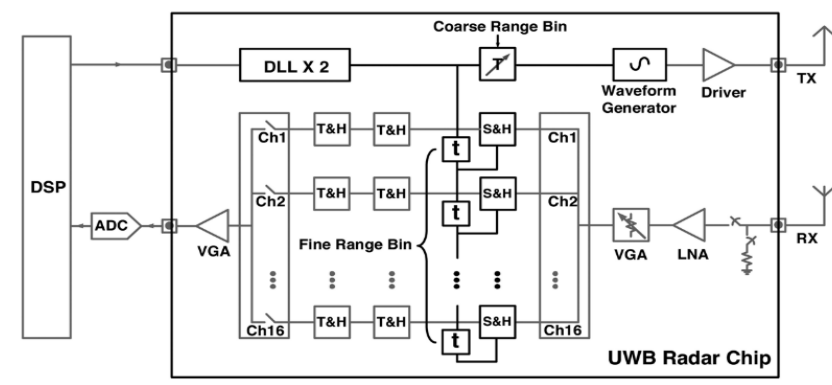

Figure 11 CMOS UWB radar diagram [45] @ 2016 IEEE

In [47], a new method using UWB impulse radar to detect human heart signal is proposed. This method is aimed at introducing a solution with low power consumption, low implementation complexity and considers the safety of the target. Spectral analysis is performed to minimize the effects of unwanted noise originating from movements of the human body on the detection accuracy. The experimental validation included a subject imitating chest vibration to ensure repeatability and reproducibility of the data. Next, an analysis on the extraction of human vital signs in the presence of noise was presented in [48]. The heart rate and respiration rate were obtained after phase demodulation of the received signal. The results show that monitoring of heart rate and respiration rate can be achieved even at $10 \mathrm{~m}$ distance from the radar.

As summarized in Table 5, various frequency bands have been used for UWB impulse radars. Several examples are the one used by [50] operating from 0.5 to $5.5 \mathrm{GHz}$, the radar in [45] with center frequencies ranging from $1 \mathrm{GHz}$ to $18 \mathrm{GHz}$, and bandwidth ranging from 7.3 to $12.7 \mathrm{GHz}$; and the radar in [21] with a center frequency of $3 \mathrm{GHz}$ and a bandwidth of 1.5 $\mathrm{GHz}$. Other reported UWB impulse radars operated in a single frequency such as in [48] using $5.4 \mathrm{GHz}$, the radar in [40] using
$5.75 \mathrm{GHz}$, and the radar in [36] using $6 \mathrm{GHz}$. In literature, the highest center frequency reported for such type of radar is at 7.3 $\mathrm{GHz}$, operating with a bandwidth of $1.4 \mathrm{GHz}$ [46].

\section{FMCW Radar}

FMCW radars are designed to overcome the disadvantages of CW and UWB radars. Operation of the FMCW radar includes the linear frequency sweeping of the transmit signal over time for repeated periods. The resulting frequency modulated signal is called a "chirp". The radar then measures the frequency differences between the transmitted signal (which is linearly frequency modulated) and the received signal from which information about the range and velocity can be obtained [2],[8]. In a recent work in [7], an FMCW radar is used to obtain the RR and HR of a patient in a bedroom environment. A signal-processing algorithm featuring advanced phase unwrapping manipulation was used. Meanwhile, the work in [51] prototyped a $60 \mathrm{GHz}$ FMCW single chip human sensing radar. Aimed at integration and size compactness, validations of this radar were performed on a target located $1 \mathrm{~m}$ away in different scenarios. Next, the work in [52] proposed a digital closed loop compensation architecture for LFMCW. It is aimed at solving nonlinear distortions caused by the analogue modules, which deteriorates detection accuracy. Despite not being specifically developed for vital sign detection, such design can be adapted for such purpose, especially when implemented using an FPGA. Besides that, a pulse noise generator is proposed to be implemented to jam a linear frequency modulated pulse compression (LFM-PC) radar in [53]. Finally, a new signal processing module and algorithm for target detection is introduced and implemented on a FPGA on a FMCW radar in [54]. It is capable to be used to extract range, Doppler speed and angle of the targets.

The authors in [60] and [16] proposed a novel chip-based radar for vital sign detection fabricated using the $40 \mathrm{~nm}$ CMOS process technology. In this work, the FMCW radar operated based on burst chirp, which is generated using a digitally controlled oscillator (DCO) structure with embedded domino chirp generation. A time domain digital predistortion block is included in the DCO to generate fast and linear chirps. Each block is controlled by the finite state machine (FSM). The radar has fat chirp slope of $0.7 \mathrm{GHz} / 40 \mu$ s with low RMS error of $0.5 \mathrm{MHz}$. Due to the deactivation of all radar circuits out of the burst-chirp duration, the power consumption was reduced more than 30 times to a record-low of $680 \mu \mathrm{W}$. This radar has the capability of detecting human respiration at a distance of $15 \mathrm{~m}$ and heart beat detection at a distance of $5 \mathrm{~m}$. The authors in [61] demonstrated the application of this chip on multi-people tracking and vital sign detection. The frequency used in FMCW radars ranges from an initial frequency and is then swept over a period of time to arrive at the final frequency. This process is repeated over multiple periods. Next, as illustrated in Table 5, a radar for detection applications is designed with a center frequency of $600 \mathrm{MHz}$ and a bandwidth of $300 \mathrm{MHz}$ in [52]. These frequencies will possibly be too low if a higher range resolution is needed, especially when potentially detecting the small movements from the chest wall. The radar used for RR 
and HR detection in [7] operates between $76 \mathrm{GHz}$ and $81 \mathrm{GHz}$, offering a high range resolution. Other operating frequencies include from as low as several gigahertz to as high as nearly $100 \mathrm{GHz}$ [8], [51], [53].

\section{SFCW Radar}

SFCW operates by stepping the frequencies in the transmit signal, or by stepping randomly selected frequencies. It can function approximately as an UWB radar in the frequency domain, and therefore has similar capabilities. Due to the frequency step, compressive sensing can be applied to this radar, which enables faster detection. In addition to that, such radars do not require high sampling rate from their ADCs. SFCW radars are also capable of target tracking and multiple targets' detection. It also has higher SNR compared to the UWB radar. In comparison to FMCW, calibration of the signal distortion due to hardware imperfections is simpler in SFCW radars. However, its main drawback is that the data acquisition time to step over many frequencies is very high, and compressive sensing algorithms are generally used to speed up this process [2].

The research in [2] uses two types of radars, UWB and $\mathrm{SFCW}$. Despite the higher resolution range-time-frequency information featured by the UWB radar, it suffers from low SNR. To overcome this, a phase-based method to tackle the issue of low SNR during human vital sign detection is proposed in [2] and [49]. The work in [2] solves the issue of SFCW long data acqusition time. It is done by proposing a multi-channel SFCW and using compressive sensing to randomly step only through $20 \%$ of the original frequencies. The block diagrams of both types of radars are shown in Figure 12 and Figure 13, respectively.

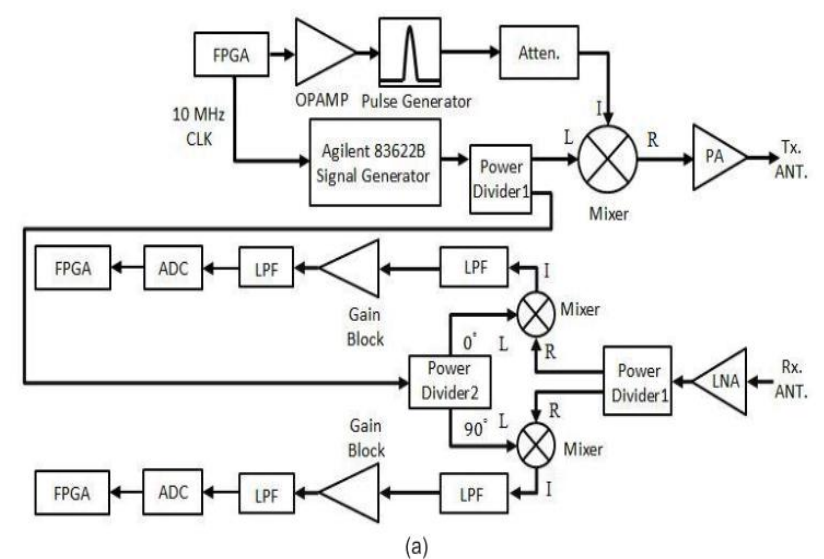

Figure 12 UWB radar system [2] and [49] (C) 2016 IEEE

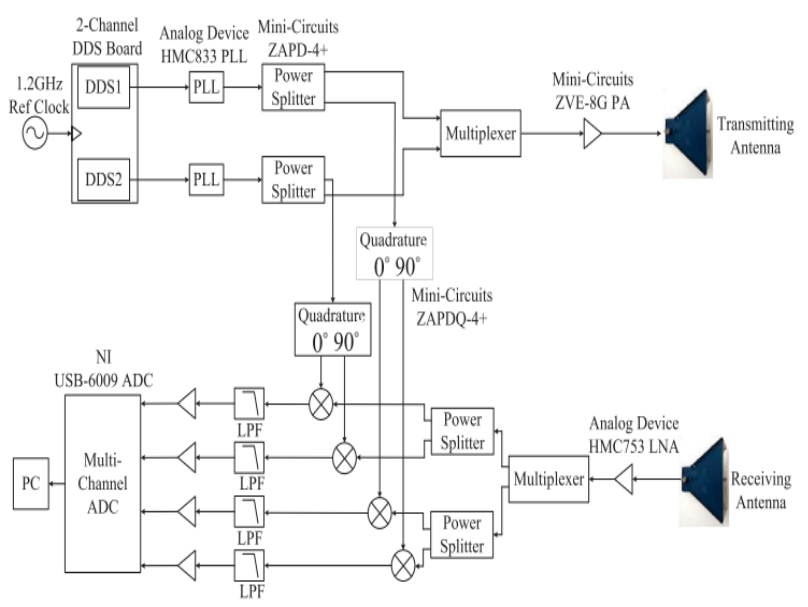

Figure 13 Multi-channel SFCW radar in [2]

Next, researchers in [9] presented the detection of human heart and breathing signal using SFCW radar. In this study, a hybrid approach of inhomogeneous object to calculate the received signal from the human rib cage and heart was employed. After that, Fourier analysis was conducted to find heart rate and respiration rate. The preliminary results show good agreement with practical data. In [55], a MIMO SFCW radar was designed to detect multiple humans via their vital signs. A signal model of the vital signs was developed first, followed by the detection method involving improving the SNR. This is prior to the application of an enhanced imaging algorithm to suppress clutter and mutual coupling. The proposed radar configuration is shown in Figure 14.

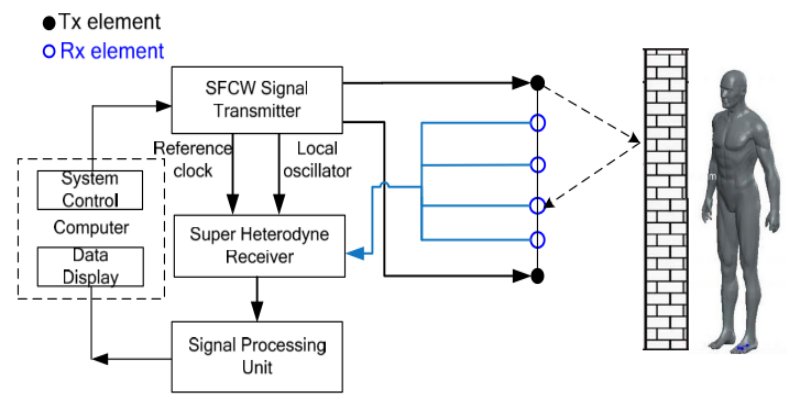

Figure 14 MIMO SFCW radar in [55]. Licensed under Creative Commons attribution license https://creativecommons.org/licenses/by/4.0/

The research in [56] studied the detection of human signals behind walls using SFCW radar. The main issue with this scenario is the substantial loss of signal energy due to wall reflections. Thus, clutter reduction methods were used to improve the detection accuracy of the vital signs. On the other hand, the effects of different human orientations and multiple humans in the environment were studied using an SFCW radar in [57]. The human rib cage model was adopted in this study. Finally, [58] presents an overview of the use of different radar types for vital sign detection. It also discusses the results of using SFCW radar, relative to reference measurements. The designed SFCW is comprised of direct digital synthesizer (DDS), controlled by a complex programmable logic device 
Table 5 Classification of literature based on the operating frequency spectrum

\begin{tabular}{|c|c|c|c|}
\hline $\begin{array}{c}\text { Radar } \\
\text { Type }\end{array}$ & Frequency Spectrum & Reference & Vital sign/other application \\
\hline \multirow[t]{13}{*}{ CW } & $2 \mathrm{GHz}$ & [32] & RR HR \\
\hline & \multirow[t]{3}{*}{$2.4 \mathrm{GHz}$} & {$[1],[20],[28]$} & RR HR \\
\hline & & [35] & RR \\
\hline & & [8] & Det. and class. of micro unmanned aerial system \\
\hline & $2.45 \mathrm{GHz}$ & {$[31]$} & RR HR \\
\hline & $3 \mathrm{GHz}$ & {$[21]$} & $\mathrm{RR}$ \\
\hline & $5.8 \mathrm{GHz}$ & {$[20],[22],[33]$} & RR HR \\
\hline & $10 \mathrm{GHz}, 16 \mathrm{GHz}$ & {$[20]$} & RR HR \\
\hline & \multirow[t]{3}{*}{$24 \mathrm{GHz}$} & {$[23],[27],[29]$} & RR HR \\
\hline & & {$[30]$} & RR \\
\hline & & {$[26]$} & HR \\
\hline & $60 \mathrm{GHz}$ & {$[3],[20],[34]$} & RR HR \\
\hline & $110 \mathrm{GHz}$ & {$[25]$} & RR HR \\
\hline \multirow[t]{6}{*}{ FMCW } & $f \mathrm{c}=600 \mathrm{MHz}, \mathrm{BW}=300 \mathrm{MHz}$ & {$[52]$} & General radar detection \\
\hline & $1-1.2 \mathrm{GHz}$ & {$[8]$} & Det. and class. of micro unmanned aerial system \\
\hline & $f \mathrm{c}=3.0085 \mathrm{GHz}, B \mathrm{~W}=15 \mathrm{MHz}$ & {$[53]$} & Noise jamming \\
\hline & $57.12-64.08 \mathrm{GHz}$ & {$[51]$} & RR HR \\
\hline & $76-81 \mathrm{GHz}$ & [7] & RR HR \\
\hline & - & {$[54]$} & General range \& velocity detection \\
\hline \multirow[t]{5}{*}{ SFCW } & $40-4400 \mathrm{MHz}$ & {$[55]$} & RR \\
\hline & $1-3 \mathrm{GHz}$ & {$[56]$} & $\mathrm{RR}$ \\
\hline & $2-3 \mathrm{GHz}$ & {$[57]$} & RR HR \\
\hline & $2-4 \mathrm{GHz}$ & {$[2],[58]$} & RR HR \\
\hline & - & [9] & RR HR \\
\hline \multirow{17}{*}{$\begin{array}{l}\text { UWB } \\
\text { impulse }\end{array}$} & $0.5-5.5 \mathrm{GHz}$ & {$[50]$} & RR HR \\
\hline & $f \mathrm{c}=1-18 \mathrm{GHz}, B W=7.3-12.7 \mathrm{GHz}$ & {$[45]$} & $\mathrm{RR}$ \\
\hline & $1.5-4.5 \mathrm{GHz}$ & {$[2],[49]$} & RR HR \\
\hline & $f \mathrm{c}=3 \mathrm{GHz}, B W=1.5 \mathrm{GHz}$ & {$[21]$} & $\mathrm{RR}$ \\
\hline & $3.3-5.3 \mathrm{GHz}$ & {$[10]$} & RR HR \\
\hline & $f \mathrm{c}=3.8-9 \mathrm{GHz}, B W=2 \mathrm{GHz}$ & {$[42]$} & RR HR \\
\hline & $f \mathrm{c}=4.5-9.5 \mathrm{GHz}, B W=1.75 \mathrm{GHz}$ & {$[39]$} & $\mathrm{RR}$ \\
\hline & $5.4 \mathrm{GHz}$ & {$[48]$} & RR HR \\
\hline & $5.755 \mathrm{GHz}$ & [40] & Displacement and vibration \\
\hline & $6 \mathrm{GHz}$ & {$[36]$} & RR HR -19 \\
\hline & \multirow[t]{2}{*}{$f \mathrm{c}=6.8 \mathrm{GHz}, B W=2.3 \mathrm{GHz}$} & {$[38]$} & RR HR \\
\hline & & {$[41],[47]$} & HR \\
\hline & $f \mathrm{c}=7.29 \& 8.748 \mathrm{GHz}$ & {$[4]$} & RR HR \\
\hline & $f \mathrm{c}=7.29 \mathrm{GHz}, B W=1.5 \mathrm{GHz}$ & [37] & RR HR \\
\hline & $f \mathrm{c}=7.3 \mathrm{GHz}, B W=1.4 \mathrm{GHz}$ & {$[46]$} & RR HR \\
\hline & - & {$[44]$} & RR \\
\hline & - & {$[43]$} & - \\
\hline
\end{tabular}

(CPLD), phased locked loop (PLL) and a power amplifier feeding the transmit antenna. At the receiver side, the antenna is connected to an LNA and an IQ demodulator. This radar has two channels to minimize acquisition time and more channels can be added with one master clock. The results indicate errors of $0.1 \%, 0.3 \%$, and $0.8 \%$ for RR of a person at $1 \mathrm{~m}, 1.5 \mathrm{~m}, 2 \mathrm{~m}$ distances, respectively. For HR results, the error was $0.4 \%$, $0.1 \%$ and $0.4 \%$, respectively.

SFCW radar uses a single tone in its transmitted signal, stepped in (sometimes random) frequency steps. This is the 
reason why such radar eliminates the need for ADC with high sampling frequency. The typical bandwidth for the SFCW radar ranges between $1 \mathrm{GHz}$ and $3 \mathrm{GHz}$ [56], $2 \mathrm{GHz}$ and $3 \mathrm{GHz}$ [57], and $2 \mathrm{GHz}$ and $4 \mathrm{GHz}$ [2], [58] as illustrated in Table 5.

In this section, a review of state-of-the-art research on commonly used types of radars for vital sign detection was presented. The CW, UWB, FMCW, SFCW have all been used in this application, and each radar has its own advantages and disadvantages. The selection of the radar type in the research is subject to practical and detection environment requirements.

The different signal processing algorithms used in the literature in the human vital sign detection application will also be discussed in the next section. The development/selection of the right algorithm can affect the detection from many angles such as detection accuracy, speed, implementation complexity etc.

\section{Signal Processing Algorithms for Vital Signs DETECTION}

Algorithms adopted in vital signs detection to process and extract useful information, depend on their objectives and vary in complexity. The challenge that needs to be addressed with the algorithms is the weak respiration and heartbeat signals, the heart signal being superimposed on the respiration signal, and the environment being filled with noise such as clutter, body movements, and other noise sources in the radar's environment. Thus, an important feature of these algorithms must be being capable of distinguishing RR from HR and distinguishing RR and HR from noise. This is crucial as abnormal RR and HR can be mistaken as noise [62].

Due to this, there have been a considerable number of algorithms introduced to "clean" the received signal from unwanted noise. Other algorithms tend to focus on increasing the SNR, since HR and RR signals are very weak. In addition to that, several other algorithms focus on the separation of the HR from the RR signal, given that the HR is much smaller than $\mathrm{RR}$. The HR is generally superimposed onto the RR signal, resulting in very sophisticated algorithms to separate and to eliminate the HR intermodulation effect on the RR signal. Therefore, most of the successfully adopted algorithms in the human vital sign detection application are computationally complex due to these stringent requirements. They mostly involve matrix inversion or multiplication, or both. Large sizes of data matrices are also involved, thus affecting the processing speed, hardware complexity, power consumption, and possibly accuracy. Such requirements also remain the main driver for researchers to develop new algorithms and detection methods, and to employ new architectures and configurations in the processing platforms.

Several algorithms for vital sign detection for radar systems have been listed and categorized in Table 6 . The multilevel fast multi pole method (MLFM) along with method of moment (MoM) algorithm were used in [31] to implemented a complex human electromagnetic model on a $\mathrm{CW}$ radar. Calculations were performed using a 13-node GPU cluster. This is aimed at accelerating the calculation process, enabling the solution of this large-sized problem. The MLFM is based on a grouping concept to speed up the iterative solution of the linear equation system of the conventional MoM. This grouping method significantly reduced the complexity of MoM from $O\left(N^{3}\right)$ to $O(N \log N)$, where $N$ is the number of unknowns corresponding to the number of edges in the meshed object. Due to the large problem size of this application, the time to complete the computation can be too long. As a result, MLFM was parallelized using high computing processor (GPU) cluster.

Another important algorithm is presented in [44] where a novel reconstruction algorithm for compressive sensing is presented for UWB radar. It is a two-stage orthogonal matching pursuit (OMP) reconstruction algorithm, designed to reduce the complexity of the reconstruction process. This algorithm included a block wise OMP estimation, weight updating and decision mechanism, and finally, fine estimation. The flow chart of the proposed algorithm is illustrated in Figure 15.

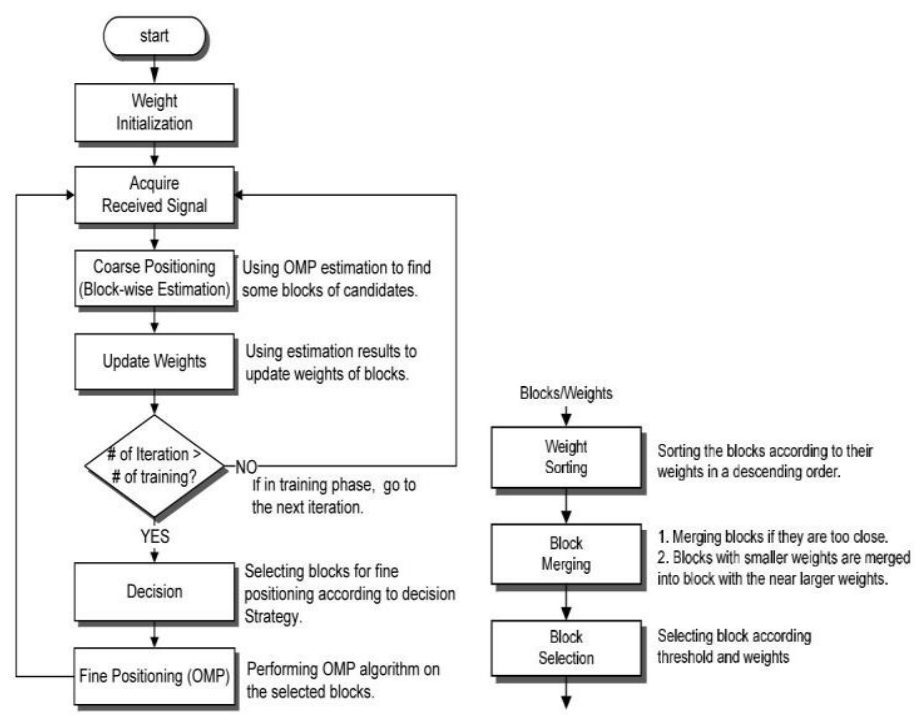

Figure 15 Two stage reconstruction algorithm [44].

Licensed under Creative Commons attribution license

The complexity of the proposed two stage OMP is much lower compared to the normal OMP algorithm. The OMP via matrix inversion bypass (MIB) algorithm can further reduce the OMP and the complexity of the two-stage algorithm, as shown in Figure 16. The reported SNR value of the radar system was $-20 \mathrm{~dB}$. 

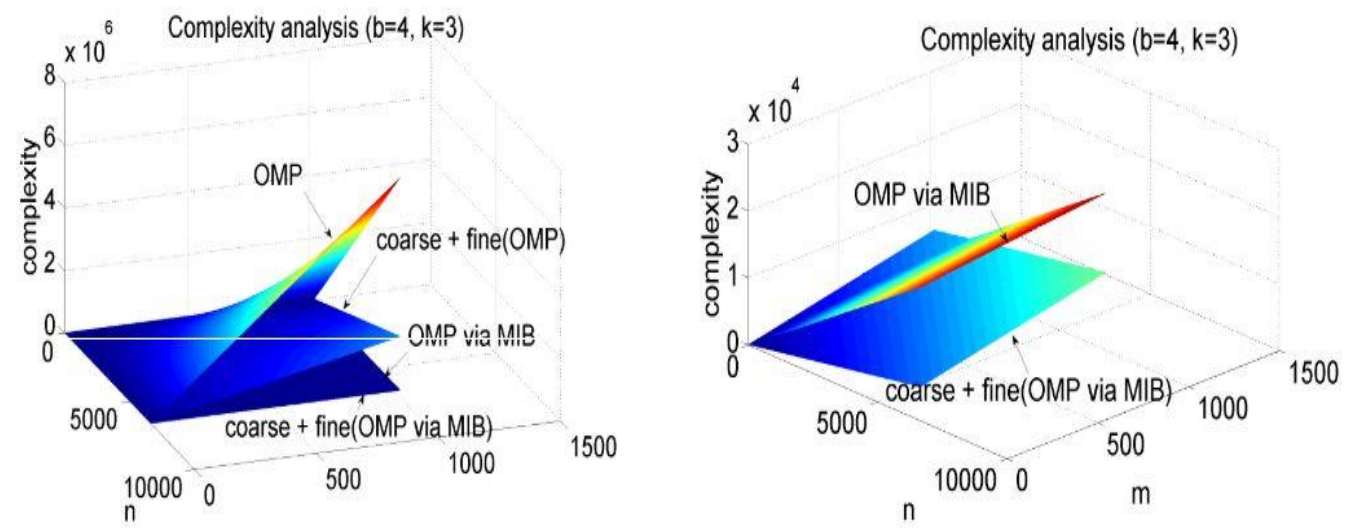

Figure 16 Computational complexity of the two-stage OMP algorithm [44]. Licensed under Creative Commons attribution license https://creativecommons.org/licenses/by/4.0/

Research on vital sign detection of single stationary target to date has progressed well. On the other hand, vital sign detection of multiple humans is still a challenge due to the mutual interference of multiple humans. A new effort to tackle this challenge was introduced in [55], in which an automatic detection algorithm which combines CFAR, morphological filtering and clustering was implemented on a UWB MIMO radar. This is intended for the vital sign detection from multiple human targets, specifically to improve the detection of weak signals reflected from them. This detection method is composed of three main procedures: preprocessing, imaging enhancements, and automatic detection and localization, as shown in Figure 17. CFAR was adopted to automatically detect multiple vital signs with large differences in magnitude in low radar cross section (RCS) environments. It uses a 2D sliding window to scan all pixels in the enhanced image to search for possible vital signs. Figure 18 is an illustrative diagram of this method.

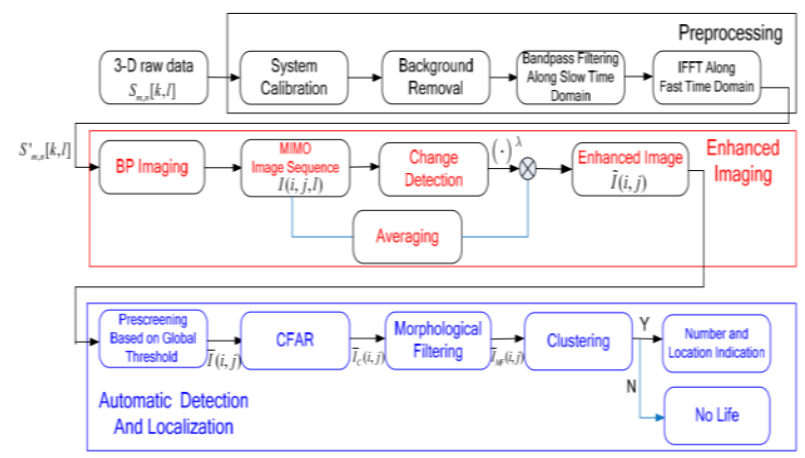

Figure 17 Automatic Detection Method [55]. Licensed under Creative Commons attribution license https://creativecommons.org/licenses/by/4.0/

One of the major problems in the vital sign detection is in scenarios where the target is behind a wall or under a wrecked building. In such scenarios, reflections due to the wall cause substantial loss of signal energy. The remaining energy signal pass through the wall and propagates towards the human target and then the weak reflected signal reaches the antenna after passing through the wall again. Due to the inevitable clutter in the radar environment, it is important that an algorithm is able to separate target signal and clutter from the received signal is introduced. In [63], the performance of the singular value decomposition (SVD) approach was compared with moving average as a clutter reduction technique in a SFCW radar. SVD, also known as the subspace method, divides the data into two categories: target and clutter subspaces. The SVD is applied on the signal matrix to separate the target signature from the clutter. This matrix can be represented as a linear combination of its eigen components. If only a single target exists, then the second eigen component contains the target information, whereas the first will contain the clutter information.

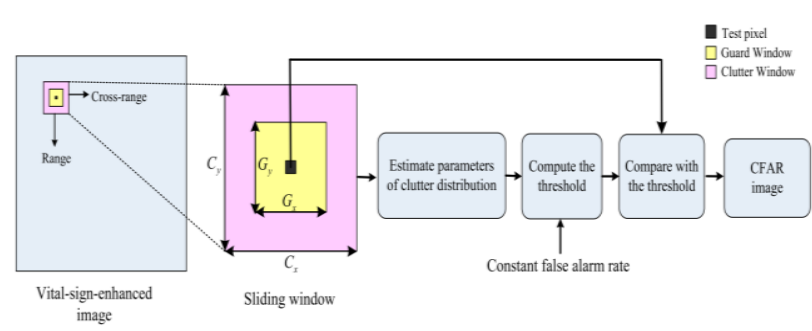

Figure 18 Illustration of the CFAR method [55]. Licensed under Creative Commons attribution license https://creativecommons.org/licenses/by/4.0/

While the chest displacement caused by breathing can be extracted from the phase variation, it is still challenging to extract the heart rate in the same way with high accuracy. This is mainly due to the harmonics of the respiration and the intermodulation between respiration and heartbeat signal that may become dominant and cause errors. To overcome this, the state space method (SSM) was applied for RR and HR detection using SFCW radar in [2]. It successfully suppressed the unwanted harmonics and intermodulation interferences in the spectrum. Experimental results for different scenarios including subject holding breath, subject breathing normally, and subject facing the radar at different angles, were reported. In all cases, the SSM successfully extracts respiration and heart rate of the 
subject. This method showed clear advantages over conventional FFT in avoiding unwanted harmonics and products resulting from intermodulation. This method also showed a relatively small error of $1.2 \%$ in the heart rate estimation. The reported SNR was $50 \mathrm{~dB}$ when combining the SSM with either CSD or AD, $46.6 \mathrm{~dB}$ when suing SSM alone compared to $8.6 \mathrm{~dB}$ when using FFT alone.

The reported algorithm in [64] uses targets removing random body motion that affect the detection of vital signs. It uses continuous wavelet transform (CWT) to identify the locations of the artifacts and then applies the moving average to smooth these identified artifacts. It also uses the discrete wavelet transform (DWT) to separate the heartbeat signal from the respiration signal which results in accurate detection. The reported process gain of this work was $32.83 \mathrm{~dB}$. Considering the reflected powers at a target at $5 \mathrm{~m}$ distance, the floor noise and the progress gain, the SNR is estimated to be $70 \mathrm{~dB}$ for the person and $53 \mathrm{~dB}$ for the chest surface.

Random body movement rejection in vital sign detection scenarios is one of the main challenges faced by the researchers. Researchers in [64] tackled this issue by first identifying the hidden in the modulated phase of the artifacts using CWT algorithm, prior to applying the moving average method to smooth the signal in those locations. Meanwhile, in [65] the features of the frequency spectrum of vital signs while undergoing random body motion are analyzed. This work utilized the motion modulation effect and extracted the direction of the body motion with the new position of the respiration peaks. Since body movements introduce frequency- shifts in the spectrum, the direction and amount of this frequency shift depends on the direction and the speed of the body motion. Thus, this feature was used to account for the body motions in the spectrum to detect the respiration rate accordingly. Meanwhile, the work in [66] effectively reduced the random movement using two methods. The complex signal demodulation (CSD) and the arctangent demodulation methods were implemented in the Doppler radar detection of vital signs. It was targeted for sleep monitoring and baby monitoring to eliminate false alarm caused by random movements. The CSD is more immune against the effects of the dc offset, whereas the $\mathrm{AD}$ reduces the effect of harmonics and intermodulation interference and high carrier frequencies. Finally, an adaptive phase compensation method was used for random body movement cancellation in [67]. To measure the random body movements of a subject, a camera was integrated in the radar system. The camera measurement was fed back into the system as the phase information. Using the phase compensation avoids potential saturation of the high gain baseband in the presence of large body movements. A simple video processing was also performed to extract the random body information without using any markers.

In this section, many of the algorithms used for vital sign detection were reviewed, regardless of the platforms on which they were implemented. Some of these algorithms focus on the rejection of clutter and noise, and thus on improving the accuracy. Meanwhile other algorithms focused on the separation of HR from RR and the extraction of the required features. Several important algorithms discussed here are the

Table 6 Comparison of Algorithms used for Vital Sign Detection

\begin{tabular}{|c|c|c|c|c|c|}
\hline & Algorithm & Purpose & Process gain/ SNR & Advantages & Disadvantages \\
\hline [2] & $\begin{array}{l}\text { SSM - complex signal } \\
\text { demod. } \\
\text { SSM - Arctangent } \\
\text { demod. }\end{array}$ & $\begin{array}{l}\text { Adopted to suppress the } \\
\text { unwanted harmonics and } \\
\text { intermodulation interferences in } \\
\text { the spectrum }\end{array}$ & $\begin{array}{l}50 \mathrm{~dB} \text { compared to } 8.4 \\
\mathrm{~dB} \text { when using FFT } \\
\text { and } 46.6 \mathrm{~dB} \text { when } \\
\text { using SSM alone. }\end{array}$ & $\begin{array}{l}\text { SSM method can improve } \\
\text { SNR by suppressing } \\
\text { harmonics. When combined } \\
\text { with other methods (such as } \\
\text { CSD and AD) it is proven to } \\
\text { achieve higher SNR values. }\end{array}$ & $\begin{array}{l}\text { The combined SSM with CSD or } \\
\text { AD algorithm is done at the } \\
\text { expense of the complexity }\end{array}$ \\
\hline [31] & MLFM and MoM & $\begin{array}{l}\text { Implementation of human } \\
\text { electromagnetic model to be } \\
\text { used for vital sign detection }\end{array}$ & N/A & $\begin{array}{l}\text { Very accurate in modeling the } \\
\text { human for vital sign detection }\end{array}$ & $\begin{array}{l}\text { Demands high computational } \\
\text { resources }\end{array}$ \\
\hline [44] & $\begin{array}{l}\text { Two stage OMP and } \\
\text { OMP via MIB }\end{array}$ & $\begin{array}{l}\text { Designed to reduce the } \\
\text { complexity of the reconstruction } \\
\text { process of compressive sensing }\end{array}$ & $20 \mathrm{~dB}$ & $\begin{array}{l}\text { Less complex than traditional } \\
\text { OMP, advantageous in } \\
\text { reducing computational } \\
\text { complexity }\end{array}$ & $\begin{array}{l}\text { Needs to be implemented in more } \\
\text { practical scenarios }\end{array}$ \\
\hline$[55]$ & $\begin{array}{l}\text { Combined CFAR and } \\
\text { morphological filtering } \\
\text { and clustering }\end{array}$ & $\begin{array}{l}\text { Designed for the detection of } \\
\text { multiple humans, specifically to } \\
\text { improve the detection of weak } \\
\text { signals reflected from human } \\
\text { targets }\end{array}$ & $\mathrm{N} / \mathrm{A}$ & $\begin{array}{l}\text { Proven in detection of } \\
\text { multiple vital sign detection } \\
\text { with large magnitude } \\
\text { difference in low signal to } \\
\text { clutter ratio scenarios }\end{array}$ & $\begin{array}{l}\text { Implemented on stationary targets, } \\
\text { more practical scenarios need to } \\
\text { be tested. }\end{array}$ \\
\hline [63] & $\begin{array}{l}\text { SVD and moving } \\
\text { average }\end{array}$ & Adopted for clutter reduction & N/A & $\begin{array}{l}\text { Proven for clutter reduction } \\
\text { from received signal where it } \\
\text { divides the data into target and } \\
\text { clutter subspaces. }\end{array}$ & $\begin{array}{l}\text { Computationally expensive and } \\
\text { slow. SVDs require care dealing } \\
\text { with missing data. }\end{array}$ \\
\hline [64] & $\begin{array}{l}\text { Random body motion } \\
\text { rejection algorithm }\end{array}$ & $\begin{array}{l}\text { Designed for suppressing } \\
\text { random body movement and } \\
\text { separating HR from RR }\end{array}$ & $\begin{array}{l}\text { Process gain } 32.83 \mathrm{~dB} \\
\text { SNR is } 70 \mathrm{~dB} \text { and } 56 \mathrm{~dB} \\
\text { for person and chest } \\
\text { surface, respectively }\end{array}$ & $\begin{array}{l}\text { The combination of CWT, } \\
\text { linear demodulation, DWT } \\
\text { techniques are proven in } \\
\text { suppressing random } \\
\text { movement and providing } \\
\text { accurate results. }\end{array}$ & $\begin{array}{l}\text { Combination of the algorithms is } \\
\text { high in complexity as it involves } \\
\text { many steps. }\end{array}$ \\
\hline
\end{tabular}


orthogonal matching pursuit, compressive sensing, singular value decomposition, and state space method. Another important aspect in radar detection for human vital sign, is the selection of processing platform. Summary of the algorithms, process gain/SNR feature and some remarks of their advantages and drawbacks have been listed in Table 6. The different processing platforms used in the literature will be discussed in the next section. The discussion will have special focus on the FPGA as a processing platform.

\section{Processing Platforms for Vital Sign Detection}

Several signal-processing platforms have been adopted for human vital sign detection. The most common platforms are: Central processing unit (CPU) of a PC, digital signal processor (DSP) unit, Graphic processing unit (GPU), application specific integrated circuits (ASIC) based processors, and field programmable gate array (FPGA). Besides these processing devices, the algorithm used also determines the processing speed, cost, resources utilization, and accuracy.

When real-time processing is not required, the computational analysis can be performed on a CPU. On the contrary, when real-time processing is required, it will be challenging for the CPU to meet requirements such as high throughput, low latency, low resources utilization, and low power consumption. To overcome those challenges for radar based real time human vital signs detection, dedicated hardware implementations, such as ASIC or FPGA have been chosen by researchers in literature [68].

Another factor in considering the type of processing platform is whether it will be used for simultaneous control and/or processing. If the processing is performed using a PC platform, then the algorithms are coded using software such as MATLAB or LabVIEW and are executed in the CPU. A separate set of tools and software is used when the processing is performed on an FPGA using hardware description languages such as VHDL or Verilog. Table 7 summarizes the state-of-the-art signal processing platforms for detecting human vital signals.

The choice of hardware (such as DSP boards, GPUs, microcontrollers or FPGAs) and consequently, the overall system's implementation costs, are dependent on factors such as the radar architecture, detection techniques chosen, and whether these techniques and/or algorithms require parallel or serial processing. Moreover, factors such as the hardware functionalities (and limitations) and the number of hardware to fully and efficiently implement the intended system functionalities also need to be considered for cost-efficiency. Their relative per unit costs are typically low to high, listed in the following order: microcontrollers, DSP boards, FPGAs and GPUs. It is also noted that product range in each of the hardware exists with varying capabilities and subranges in costs. In many instances, FPGA's are used as verifications tool, whereas in other instances, depending on the application, they also can be used as central processing unit. This can be tied to the cost effectiveness, for example, by using a single central processing unit instead of linking each radar with as separate processor to lower the cost of implementing the overall system.
In [51], a DSP and microcontroller unit were used as processing platforms, while in [29], a microcontroller is used for digitization and a $\mathrm{PC}$ is used for further processing. Besides this, the work in [4] is performed based on SoC and PC processing, while [69] is based on FPGA and GPU processing. Moreover in [30], only a DSP core is used, and in [42] only an $\mathrm{SoC}$ is used. As can be seen from Table 7, vital sign detection can be performed on hybrid platforms, or on a single processor type, such as FPGA, PC, or DSP. The table also classifies the researches in which FPGA was applied, into two categories: (i) radar with FPGA used as processing platform, and solution is specifically targeted for vital signs detection; or (ii) radar with FPGA used as processing platform, but its application is not specifically targeted for vital signs detection. However, for the latter, the FPGA architecture and implementation can be modified or extended for application in vital signs detection. The classification of literature based on the processing platform is particularly useful when determining specific platforms for the implementation of different algorithms.

Table 7 Summary of radar and/or vital sign detection processing platforms in literature

\begin{tabular}{|c|c|}
\hline Platform & Reference \\
\hline $\mathrm{PC}$ & $\begin{array}{l}3],[20],[22],[23],[25],[26],[28],[37],[35], \\
{[7],[38],[39],[55],[56],[57],[70],[71]}\end{array}$ \\
\hline FPGA & $\begin{array}{l}{[2],[21],[31],[54],[40],[43],[44],[45],[49],} \\
{[62],[72],[73],[74],[75],[76]}\end{array}$ \\
\hline $\begin{array}{l}\text { FPGA (not specifically } \\
\text { for vital sign detection) }\end{array}$ & $\begin{array}{l}\text { [52], [53], [68], [77], [78], [79], [80], [81], } \\
{[82],[83],[84]}\end{array}$ \\
\hline SoC & {$[42]$} \\
\hline DSP & [30] \\
\hline FPGA-GPU & [69] \\
\hline PC-microcontroller & {$[29],[27]$} \\
\hline DSP-microcontroller & {$[51]$} \\
\hline SoC-PC & {$[4],[1]$} \\
\hline $\begin{array}{l}\text { No focus on the } \\
\text { processor }\end{array}$ & $\begin{array}{l}{[8],[9],[10],[32],[33],[34],[46],[47],[48],} \\
{[50]}\end{array}$ \\
\hline
\end{tabular}

FPGA has been widely used to implement algorithms on hardware, to confirm their accuracy and to ensure effective real time analyses [62]. The reconfigurable nature of an FPGA offers a multifunction implementation, resulting in resource efficiency compared to separate implementations of functions on an ASIC-based processing platform, for instance. In addition to that, its cost-efficiency and reconfigurability makes it a preferred rapid prototyping platform for researchers [69].

The use of FPGA as a processing platform in the area of radar systems has been increasing steadily. Table 8 summarizes the 
state-of-the-art literature where FPGA is used as a control/processing platform within a radar-based system for detection and monitoring of HR and RR signals. FPGAs were used for signal pre-processing, interfacing, as well as synthesis of certain mandatory blocks. Besides that, it can also be applied for control, digitization, signal generation, filtering, and demodulation. Another important function of an FPGA is to serve as an implementation platform of novel algorithms for HR and RR detection and monitoring. A summary of these algorithms implemented on FPGA is also presented in Table 8. Many of these articles presented novel FPGA designs and architectures to implement these algorithms. Some of these algorithms have been specifically implemented on FPGA for vital sign detection and monitoring, while others have been developed for other applications. However, most of them can be used for human detection with minor modifications. The features of flexibility and reconfigurability in FPGAs have influenced designers to apply them not only as a verification tool. Unlike ASICs, FPGAs can be used as an external processor due to its better flexibility in reconfiguring its design after implementation.

These FPGA-based implementations have been applied to different types of radars such as CW, FMCW, UWB, and SFCW radar. More specifically, FPGA has been used in the context of CW radar in [21] and [31]. For UWB radar type, FPGA has been used in [21], [45] and [49]. Other pulse or impulse based radar also used FPGA such as in [83], [53], [44] and [40]. Other state-of-the-art articles in the table do not report the specific type of radar where the reported synthesis/implementation of the algorithm should be applied to.

The different functions of FPGA in the literature have led to improved designs and validated implementation in the detection and monitoring of HR and RR signals. Whenever FPGA is adopted as the main platform for pre-processing or processing platform to extract $\mathrm{RR}$ and/or $\mathrm{HR}$ information, certain parameters have been typically focused on. These include the logic utilization, processing time/speed, latency, and accuracy by capitalizing on its parallelism ability.

In [21], an FPGA was used to synthesize the digital downconverter as well as for sampling and digitization in the receiver part of the CW radar. Meanwhile, in the UWB radar, the main use of FPGA is for generating the clock frequency. Further processing of the UWB signal was performed using PC, and FPGA was also used as interface and control module, and as temporary memory of digitized data. Further improvement on this work was performed by implementing the Doppler frequency extraction algorithm (STFT) on both radar types (CW and UWB). In [74], an FPGA was used to synthesize the binary phase shift keying modulator/demodulator, which is used for high range resolution detection. This implementation achieved a processing delay of around $2.569 \mathrm{~ns}$. Besides that, reconfigurable phase shift keying was synthesized using FPGA in reference [79].

An OMP algorithm was used for CS reconstruction and was implemented on FPGA in [68] with a frequency of $165 \mathrm{MHz}$ and dictionary size 512 × 2048 . This implementation achieved a 33-time increase in speed compared with previous designs, and the time required for construction is around $391.8 \mu$ s with
$1.2 \times 10^{-3}$ accuracy. An illustration of the FPGA implementation and interface of the algorithm and the design architecture is shown below.

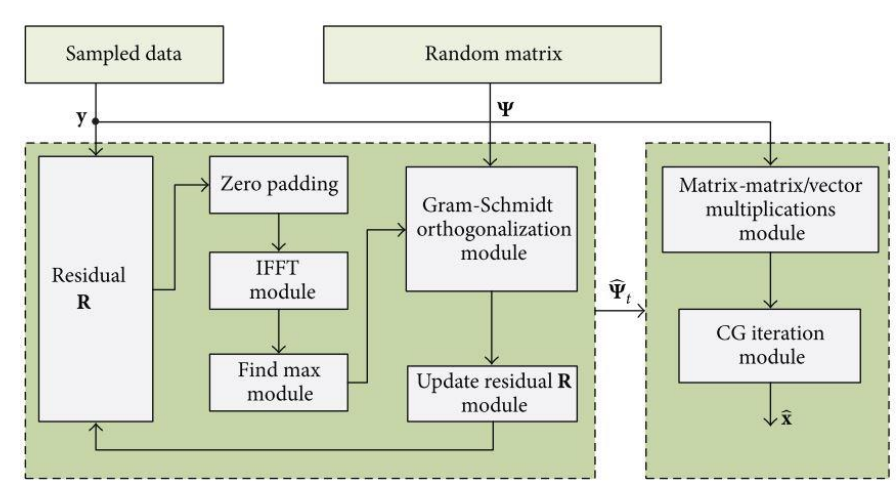

Figure 19 Architecture of FPGA top level entity implementation [68]. Licensed under Creative Commons attribution license https://creativecommons.org/licenses/by/4.0/

For the LFMCW in [52], a DDS algorithm combined with CORDIC algorithm was used to build its nonlinear distortion compensation scheme, implemented on an FPGA. This implementation improved the peak side-lobe-ratio (PSLR) from $5.7 \mathrm{~dB}$ to $0.3 \mathrm{~dB}$ after applying the scheme. Next, the MPSO and HAS algorithms implementation in [77] was performed on FPGA for radar applications. Another algorithm implementation was developed and implemented on FPGA/GPU in [69]. The new CS reconstruction algorithm is called blocking compressive sampling matching pursuit (BCoSaMP). This work also introduced a signal-processing tool (RSPT) which allowed designers to auto-generate fully optimized VHDL representation of BCoSaMP by just specifying several parameters. The reported FPGA implementation achieved 14-times faster processing over the sequential implementation, while the GPU implementation achieved a 10.7-time speed-up. For samples of 256, 512 and 1024, the throughput was 41025, 71793 and 116304 cycles, respectively, whereas the execution time was $0.3487 \mathrm{~ms}, 0.61$ $\mathrm{ms}$ and $0.988 \mathrm{~ms}$, respectively. The reported power consumption was $1960 \mathrm{~mW}$. For 512 samples, the reported execution time was $3.1 \mathrm{~ms}, 0.3487 \mathrm{~ms}$, and $0.442 \mathrm{~ms}$ for sequential, FPGA, and GPU processing, respectively.

Another DDS implementation using FPGA was reported in [78]. This DDS has quadrature outputs, is based on look up tables (LUT), and was designed with a $100 \mathrm{MHz}$ system clock. This design and implementation have spurious free dynamic range (SFDR) of $114 \mathrm{~dB}$, which was improved by $70 \mathrm{~dB}$ compared to traditional DDS. However, this was at the expense of increased logic utilization. Next, a digital pulse compression algorithm was developed and implemented on FPGA in [80]. The use of FFT-OS method to provide range resolution and to improve the SNR was used in the DPC. The design achieved a pulse compression gain of $27 \mathrm{~dB}$ and peak range side lobe ratio of $-56 \mathrm{~dB}$. Meanwhile, a linear frequency modulation with 3 $\mu$ sec pulse duration using an off the shelf FPGA based DDS was designed in [81]. The design and implementation of the LFM 
consisted of a) Implementation of spot frequency generation (from $150 \mathrm{MHz}$ to $350 \mathrm{MHz}$ ) using DDS AD9858; b) Design and realization of LFM waveform using DDS for higher bandwidth. Simulation and results for a bandwidth of up to 200 $\mathrm{MHz}$ were reported. In [82], a real time waveform generator suited for wideband and supporting many popular modulation schemes, was reported with its FPGA implementation. This implementation was reported with a DAC capable of up to 2.5 GSps and with reduced memory manipulation to change waveform by two to three orders of magnitude. The reconfiguration time was reported to be in the range of hundreds of nano seconds, and the logic utilization was less than $5 \%$ of modern FPGA resources. As an example, for the $\mathrm{CW}$, its memory requirement is 21.625 bytes and reconfiguration time is $120 \mathrm{~ns}$.

In [83], a fast convolution processing-based pulse compression and pulse Doppler processing were presented and implemented on FPGA. It resulted in improved target detection, range resolution, and speed estimation. Meanwhile, the work in [53] presents the FPGA implementation of a noise jammer using the pulsed noise jamming technique. The detection capability of the radar was reduced as the jamming to noise ratio (JSR) is increased, and the radar was blind at a JSR of $30 \mathrm{~dB}$. Another reconfigurable modulator was reported and implemented in [84]. The FPGA implementation was reported to be dynamically reconfigurable on the fly, and features between $10.2 \%$ and $91.43 \%$ hardware resources utilization, and $76.38 \%$ of power consumption reduction. This is relative to the conventional non-reconfigurable modulator design. The reconfiguration time requirement for amplitude modulation and frequency modulation was reported to be $121 \mu \mathrm{s}$, whereas for other modulation types, reconfiguration time takes $184 \mu \mathrm{s}$.

In [54], an FMCW radar for target detection via FFT was designed. To alleviate the problem of constant false alarm rate (CFAR), an ego-velocity compensation algorithm was introduced and implemented on an FPGA. The total processing time reported was $60 \mathrm{~ms}$ with $97 \%$ reduction in CFAR upon the implementation of the algorithm. The ego-velocity compensation logic reduction was decreased by around $96 \%$, whereas in the case of CFAR, it was reduced by $62 \%$. Meanwhile, a series of radar signal processing algorithms called adaptive pulse compression, and specifically the least square estimator, were implemented on FPGA with coprocessor in [75]. Implementation was performed using different architectures; (i) pipelining architecture, with an improved latency, but at the expense of high logic utilization; (ii) distributed memory architecture, which also has high logic utilization with better latency. This optimization architecture was reported to have reduced interval initiation of the coprocessor by two, but increased hardware utilization by 1.5 times, while at the same time, reduced latency of the LS matrix when larger than $16 \times 16$.

A CMOS impulse radar with two stage reconstruction algorithm for CS, and implemented on a FPGA, for human respiration feature extraction was reported in [44]. This algorithm featured $75 \%$ less complexity compared with the OMP algorithm for CS reconstruction. When the implementation was designed with a $318 \mathrm{MHz}$ clock, a radar image resolution of $256 \times 13$ was achieved, with a throughput (radar image rate) of 28.2 frames per second and a latency of
$35.4 \mathrm{~ms}$. Figure 20 shows the architectural blocks used for implementing the proposed OMP.

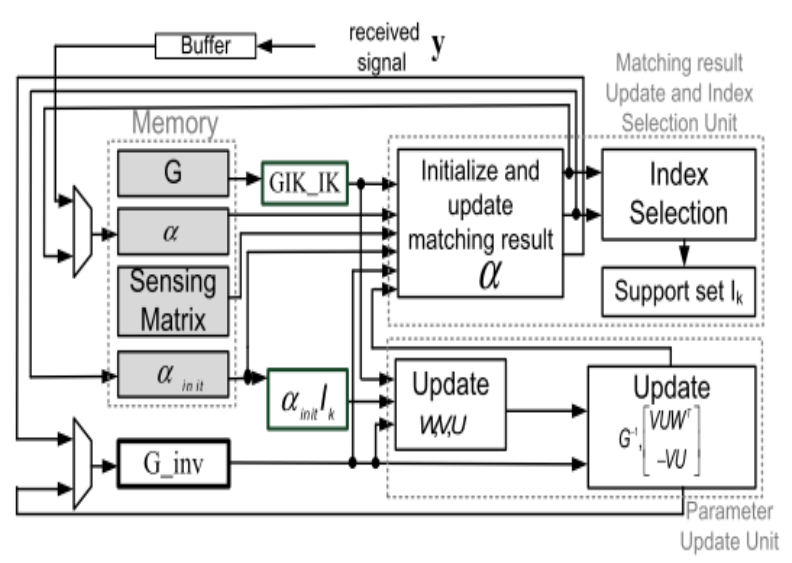

Figure 20 Architectural Block of algorithm FPGA implementation [44]. Licensed under Creative Commons attribution license https://creativecommons.org/licenses/by/4.0/

Meanwhile, another UWB radar and a respiration model, called FSLW model, along with chirp-Z transform to extract RR features, was presented in [45]. The FPGA implementation of the algorithm resulted in a maximum NSME of around $3.93 \%$ for the FSLW model and around $9.67 \%$ for the MRCW model. Even though the $\mathrm{C}-\mathrm{Z}$ transform is three times more complex compared to the FFT, the frequency estimation error is higher in the latter case.

In [2] and [49], the CSD and $\mathrm{AD}$ were techniques used to improve detection accuracy of heart rate signals. These methods were combined with SSM to further increase the accuracy. The target subject was located at a distance of $0.8 \mathrm{~m}$ and the results were reported for more cases when the subject was holding its breath and when breathing normally. When the subject is holding its breath, maximum deviation of the HR was reported to be $3.1 \%$ and $1.7 \%$ using direct FFT and using SSM, respectively. However, this was minimized when using SSMCSD and SSM-AD to $0.5 \%$. The maximum SNR was reported to be 50.2 when using SSM-AD, whereas it was 8.4 when using direct FFT. In the case of the target breathing normally, the maximum deviation of HR was reported to be $3.7 \%$ when using SSM and this was minimized to $1.7 \%$ when using SSM-CSD and SSM-AD. The maximum SNR was reported to be 32.6 when using SSM-AD and was 20.4 when using AD only. The FPGA was used in this work to generate the clock frequency, to digitize the data stored, and for further processing. In [31], an electromagnetic model of a walking human and of human vital signs were developed and validated using a $\mathrm{CW}$ radar. The main function of the FPGA in this design was an interfacing chip for the control and baseband signal. Meanwhile, an MRMN adaptive filter algorithm was developed and implemented on FPGA in [76]. The post route and place implementation of this FPGA indicated nearly $90 \%$ of reduction in resource utilization and nearly 2.6 times improvement in terms of clock frequency and speed. In [40], a range compression (time-domain crosscorrelation) algorithm was developed and implemented on an 
FPGA for impulse radar. This was performed with a carrier frequency of $5.77 \mathrm{GHz}$ and transmit power of $30 \mathrm{dBm}$. The design achieved cross correlation computation time of 121.63 $\mu \mathrm{s}$ and a vibration spectrum monitoring of up to $50 \mathrm{~Hz}$.

Table 8 Summary of FPGAs Used and the Algorithms/Methods

\begin{tabular}{|c|c|c|}
\hline Ref. & Methods/Algorithms & FPGA Use \\
\hline [21] & $\begin{array}{ll}\text { STFT to obtain Doppler frequency } \\
\text { ETSM for narrow pulse digitization }\end{array}$ & $\begin{array}{l}\text { - Synthesize DDC in receiver } \\
\text { - Sampling/digitization process } \\
\text { Generating clock frequency, } \\
\text { temporary storage, control } \\
\text { and interface with PC }\end{array}$ \\
\hline [74] & $\begin{array}{l}\text { Digital modulator/demodulator } \\
\text { synthesis (BPSK modulator) }\end{array}$ & $\begin{array}{l}\text { Modulator/demodulator } \\
\text { synthesis }\end{array}$ \\
\hline [68] & $\begin{array}{l}\text { Modified OMP for CS reconstruction } \\
\text { FFT for correlation optimization } \\
\text { CG for large scale least square } \\
\text { problem }\end{array}$ & Algorithm implementation \\
\hline [52] & $\begin{array}{l}\text { DDS combined with CORDIC to build } \\
\text { a nonlinear distortion compensation } \\
\text { scheme. }\end{array}$ & $\begin{array}{l}\text { Distortion compensation } \\
\text { scheme implementation }\end{array}$ \\
\hline [77] & $\begin{array}{l}\text { GOA specifically MPSO } \\
\text { HSA for Mutation }\end{array}$ & - Algorithm implementation \\
\hline [69] & $\begin{array}{l}\text { New CS called BCoSaMP } \\
\text { RSPT }\end{array}$ & - Algorithm implementation \\
\hline [78] & $\begin{array}{l}\text { DDS based on LUT using wave } \\
\text { compression and Taylor series }\end{array}$ & DDS synthesis \\
\hline [80] & - DPC via FC using (FFT-OS) & - DPC implementation \\
\hline [81] & LFM using FPGA based DDS & DDS implementation \\
\hline [82] & Waveform generation & $\begin{array}{l}\text { Waveform generator } \\
\text { synthesis }\end{array}$ \\
\hline [83] & $\begin{array}{l}\text { FC based pulse compression and pulse } \\
\text { Doppler processing }\end{array}$ & - Algorithm implementation \\
\hline [53] & $\begin{array}{l}\text { - Noise jammer using pulsed noise } \\
\text { jamming techniques. }\end{array}$ & - Algorithm implementation \\
\hline [84] & - Reconfigurable Modulators & Modulator synthesis \\
\hline [54] & $\begin{array}{ll} & \text { FFT } \\
& \text { EFAR } \\
& \text { Ego-VC } \\
\end{array}$ & - Algorithm implementation \\
\hline [75] & - APC specifically LSE & - Algorithm implementation \\
\hline [44] & $\begin{array}{l}\text { Two-stage reconstruction for CS } \\
\text { FSLW model }\end{array}$ & Algorithm implementation \\
\hline [45] & $\begin{array}{l}\text { FSLW with early termination } \\
\text { Chirp z-transform (CZT) }\end{array}$ & Algorithm implementation \\
\hline$[2]$ & $\begin{array}{l}\text { CSD and AD combined with } \\
\text { - SSM }\end{array}$ & $\begin{array}{l}\text { Clock frequency generation, } \\
\text { digitized data storage }\end{array}$ \\
\hline [31] & $\begin{array}{l}\text { Electromagnetic model for motion and } \\
\text { vital sign } \\
\text { - MLFM and MoM }\end{array}$ & $\begin{array}{l}\text { Interfacing chip; through } \\
\text { Ethernet; of control and } \\
\text { baseband signals }\end{array}$ \\
\hline [76] & - MRMN adaptive filter & - Algorithm implementation \\
\hline [40] & $\begin{array}{l}\text { - Range compression for time-domain } \\
\text { cross-correlation }\end{array}$ & Algorithm implementation \\
\hline [79] & - Reconfigurable PSK modulator & - Modulator synthesis \\
\hline
\end{tabular}

Table Abbreviations:

STFT: Short Time Fourier Transform

ETSM: Equivalent Time Sampling Method

DDC: Digital Down Converter

BPSK: Binary Phased Shift Keying

OMP: Orthogonal Matching Pursuit

CS: Compressive Sensing
FFT: Fast Fourier Transform

CG: Conjugate Gradient

DDS: Direct Digital Synthesis

CORDIC: Coordinate Rotation Digital Computer

MPSO: Modified particle Swarm Optimization

HAS: Hamming Scan Algorithm

Blocking Compressive Sampling Matching Pursuit: BCoSaMP

RSPT: Radar Signal Processing Tool is a tool allows the designer to auto-

generate fully optimized VHDL representation of BCoSaMP

GOA: Global Optimization Algorithm

LUT: Look Up Table

DPC: Digital Pulse Compression

FC: Fast Convolution

OS: Overlap Save method

LFM: Linear Frequency Modulation

IFFT: Inverse Fast Fourier Transform

CFAR: Constant False Alarm Rate

Ego-VC: Ego-Velocity Compensation

APC: Adaptive Pulse Compression

LSE: Least Square Estimator

FSLW: Four-Segment Linear Waveform Model.

CZT: Chirp Z-Transform

CSD: Complex Signal Demodulation

AD: Arctangent Demodulation

SSM: State Space Method

MRMN: Modified Robust Mixed Norm

MLFM: Multi Level Fast Multi Pole Method

PT: Pan Tompkins Algorithm

PSK: Phase Shift Keying

MRCW: modified raised cosine waveform model

In this section, the commonly used processing platforms for vital sign detection have been reviewed. Researchers in the reviewed literature used PC, MCU, GPU, FPGA, DSP, or a combination of these platforms. The choice of the dedicated processing platform, such as FPGA, is made by these researchers to achieve higher processing and detection speeds. The different uses of FPGAs in the context of vital sign detection and the different algorithms implemented on FPGAs have also been discussed.

\section{DETECTION AND COMMUNICATION}

It is inevitable that detected signals via radars are required to be transferred/communicated in some way to another location. Several frequency bands used for communication in biomedical applications such as the industrial, scientific and medical (ISM) band, the UWB band, Radio Frequency Identification (RFID) band, Bluetooth frequency band, WLAN frequency band and Medical Body Area Network (MBAN) frequency band [20], [85]. Recently, several approaches for vital sign detection integrated with communication approaches have been proposed.

\section{A. Detection}

This subsection illustrates the techniques used for vital sign detection using communication devices (such as WLAN routers). For example, the work in [86] proposed time reversal based respiration rate detection within a very short period of time. This approach used off the shelf WLAN devices and their channel state information (CSI) to capture small variations in the surroundings caused by respiration [86],[87]. This method can be easily implemented using any existing WLAN hardware and networks available indoors. The two prototypes in [86] were built using WLAN cards with three omnidirectional 
antennas. One of the prototypes works as the access point, while the other one works as the station. The center frequency used was $5.765 \mathrm{GHz}$ with a bandwidth of $40 \mathrm{MHz}$. During experiments, only two to three WLAN networks were observed to be sharing the same channel, resulting in less than $1 \%$ of packet loss rate, which is insignificant and can be ignored. Jian et al. in [88] proposed a system to detect both heart rate and breathing during sleep using off-the-shelf WLAN (WiFi) devices. Similar to [86] and [87], this system reused the existing WLAN network and exploited the channel state information to capture the tiny movement due to respiration. This experiment was conducted in an 802.11n WLAN (Lenovo T500 Laptop) connected to a wireless access point (AP) (model TP-Link TLWDR4300) with a packet transmission rate of $20 \mathrm{pkts} / \mathrm{s}$.

Meanwhile, in [89], a ubiquitous off-the-shelf WLANenabled device was used to detect breathing using the received signal strength (RSS). This can be performed due to the introduction of a dominant periodic component in the standard WLAN received signal. The proposed system can help to reliably extract the hidden breathing signal from a noisy WLAN RSS. The system handles many challenges, including noise elimination, interfering human, sudden movements as well as abnormal breathing situations. The functionality of remote monitoring may be restricted when using the available wireless infrastructure. Once there is a wireless terminal with an RF front-end transceiver and network connection, the detection of vital signs and the communication of the collected data to a remote monitoring facility will take place. Information on human respiration and heart rate only requires low bandwidth transmission capability. In [90], Victor et al. used an add-on module to an existing wireless terminal to detect human heart and breathing activities. The module included an antenna and mixing element to receive the transmission from the wireless terminal, which then produced a Doppler-based signal proportional to the heart and chest motion. This produced signal can be used for detection of heart and breathing activities and can potentially be relayed by the wireless terminal to a remote heath monitoring facility via the existing telecommunication network and infrastructure.

\section{B. Communication}

This section illustrates the different approaches and techniques used for combined sensing and communication functionality. The main drive for the integration of radar sensing with communication is to arrive at a compact hardware solution. Components such as transceiver and antenna can serve dual functions - in sensing and in communication. These systems operate in two modes: the detection mode measures range, velocity angle, etc., whereas the communication mode receives and demodulates the spread spectrum and returns connection with a remote station. Other solutions include system solution where the frequency band of the communication transceiver is smaller compared to the pulse spectrum of the radar. This is so that both bands overlap, and the same RF front end could be used for both purposes, thus decreasing the cost of the system. This subsection also presents several designs where signals from sensor networks are transmitted wirelessly to base stations.

The research presented in [91] studied the approach of using the same UWB transceiver for both sensing and communication. This system is focused specifically on heart rate variability (HRV) and its link as an indicator for the cardiovascular nerve system. Off the shelf commercial transceivers were used with minor modifications. The higher resolution in UWB systems offers more accurate sensing, whereas its resistance to multipath is used for high speed communication. From the bio-signal types that can be measured using this approach, heartrate was selected due to its importance. In this study, UWB radar principles were used to measure to heartbeat and the UWB communication standards were used to wirelessly transmit these measurement results. Such approach with dual purpose - sensing/detection and communication, makes these devices ideal nodes for wearable computing and in body area networks.

Next, Bharat et al. in [92] highlighted the many advantages of using UWB as both a sensing and a communications standard for biomedical applications. These include its low radiated power $(-41.3 \mathrm{~dB} / \mathrm{MHz})$, low power consumption, ability to coexist well with other wireless technologies and robustness to interference and multipath. This work integrated the sensing and communication functionalities into a single device using FM-UWB, enabling it to be used in two operational modes for heart rate monitoring. It is able to collect vital signs from its sensors and transmit to other sensors or to repositories in real time. While a data rate of $240 \mathrm{kbps}$ is generally sufficient in biomedical applications, heart monitoring requires less than 100 bps. This can be easily implemented in FM-UWB technology, enabling the health data to be transmitted to a remote medical server frequently for better diagnosis or for better responsiveness to emergencies. Next, the integrated transceiver proposed in [92] includes several purpose-dedicated components. These include the FM modulator for sensing, and the FSK demodulator for communication. Common components for both purposes used are the low pass filter and low noise amplifier. Sensing in the transceiver was performed using the simultaneous multiple frequency transmission method. This involved a slight increase in the complexity of the transmitter hardware compared to a conventional transmitter, where two FM modulators are needed instead of one.

In [93], a biomedical wireless radar sensor network (BWRSN) for vital signs monitoring and fall detection was proposed. This is to overcome the limitations of using a single radar in real situations. The BWRSN consists of four radarbased sensor nodes and a base station. Each node consists of a microwave radar, a Zigbee module, and a microcontroller [93], [94]. The radar block generates and sends a CW signal at 5.8 $\mathrm{GHz}$ to the target and receives the reflected signal. The digitized baseband information is then transmitted wirelessly to the base station for remote data processing to determine the vital signs rate and fall detection incidents. The proposed BWRSN was tested in a lab with two nodes fixed to the ceiling, and the other two were on the wall as shown in Figure 21. Experimental results demonstrated the limitation of using a single sensor in real situations and the ability of BWRSN in detecting 
emergency situations while monitoring breathing.

The research in [95] described the design of a monitoring system for the purpose of baby monitoring. This system consists of a monitoring unit and a receiver unit. The monitoring unit is to be hanged on the side of the infant's crib to detect his/her breathing and heart rate. Collected signals are then communicated wirelessly to the receiver unit. Alarm sound and red lights will flash on both units if the child respiration and heart rate is detected to be too weak. The monitoring unit consists of an RF circuit to send and receive the radio signal, a microcontroller for processing, an Xbee chip for communication with the receiver, and several buttons and switches. The generated signal for transmission is a single tone at $5.8 \mathrm{GHz}$. The Xbee wireless transceiver communicates with the receiver in the monitoring unit and keeps it updated with the current alarm status. The choice of $5.8 \mathrm{GHz}$ is due to its suitability in detecting vital signs, besides being an unlicensed ISM band, with wide availability of low-cost components. This prototype is capable in detecting vital signs up to $1.15 \mathrm{~m}$, while the receiver unit can be as away as $50 \mathrm{~m}$ to be able to receive data from the monitoring unit. The block diagrams of the monitoring and receiving units are shown in Figure 22.

In situations where a large network of radars and communication devices are co-located, their co-existence in the same spectrum will increase interference and affect performance. The tradeoff of these essential parameters in such situations has yet to be investigated [96]. A concept was presented in [97], with a system that can be used as radar or as communication device. The proposed system uses off-the-shelf components with orthogonal frequency division multiplexing (OFDM) architecture. Next, the work in [98] presented a similar concept applied to drones or unmanned systems. A practical mobile imaging device utilizing the $60 \mathrm{GHz}$ band was introduced. The components for the communication were reused to image an object and perform measurements along the trajectory of this system. The authors in [99] proposed a wireless sensor network for a home environment, in which the sensors are dual mode radars for remote localization and fall detection. In this work, the network consists of multiple sensor nodes and a base station. The most important validation in the dual mode operation is that the radars' functionality will not interfere with the operation of the wireless communication module. Time division multiplexing (TDM) is adopted to ensure that the wireless communication and each radar sensor do not function at the same time. In addition to this, frequency division multiplexing (FDM) is used to minimize interference between the radars. Experimental results of this work validated the feasibility of this method in conducting real time detection without interference. In [100], a system of using a common waveform for vehicle radar as well as vehicle communication system based on the WLAN standard was proposed. Specifically, an IEEE 802.11(ad)-based radar for long-range application was designed in the $60 \mathrm{GHz}$ unlicensed band. Despite being intended for vehicle application, it illustrates the same concept of coexistence and functionality of radars and communication systems.

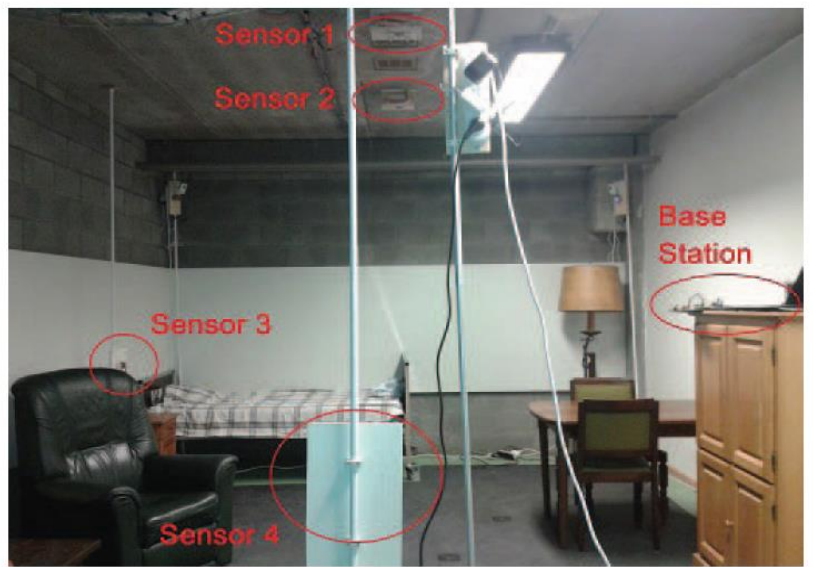

Figure 21 Sensor nodes and base station setup in [93] (C) 2016 IEEE

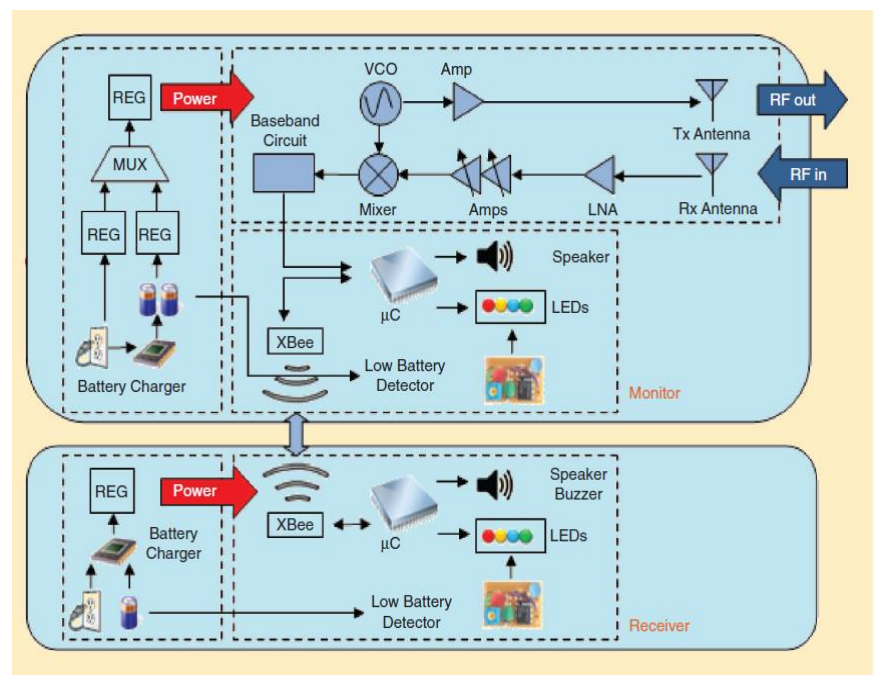

Figure 22 Block diagram of monitoring system described in [95] (C) 2009 IEEE

On the other hand, the work in [101] introduced the concept of personal mobile radar using a large number of arrays to map the environment. This radar operates in the millimeter(mm)wave band and it enables the integration of such large arrays within the users' $5 \mathrm{G}$ mobile devices. This work proposed a gridbased Bayesian mapping approach by introducing a new state space model. This research highlighted the correlation between the angular resolution, scanning time, signal bandwidth and ranging accuracy, besides methods to trade-off between these parameters. Results validated the feasibility of the introduced system concept, and a significant performance improvement in environment mapping is attained. This could be potentially attractive for applications such as indoor mapping using lowcost massive array antennas embedded in next generation smartphones. Meanwhile, realizing the spectrum scarcity, Awais et al. [102] introduced a spectrum sharing methodology. The proposed method is a spatial approach for spectrum sharing between a MIMO radar and an LTE cellular system with a number of base stations. Since the MIMO radar and LTE standard share a number of channels, an interference-channelselection algorithm is introduced. Signals from the MIMO radar were projected onto the interference channel with maximum null space. Careful selection of the interference channels 
minimizes interference from the MIMO radar and at the same time, protects the LTE base station from interference from the radar. Meanwhile, the coexistence between IEEE802.11 WLAN and radars operating in adjacent channels $(5 \mathrm{GHz})$ was studied in [103]. A modified WLAN receiver link was designed to mitigate the interference by impulse radar. Two proposed approaches for interference detection were reported, firstly the time domain cyclic prefix auto-correlation detection, and secondly, the frequency domain data subcarrier-based detection. The proposed system can significantly mitigate radar interference at high and low interference to noise ratios (INRs), whereas partial interference mitigation is also possible within the INR of $3<\mathrm{INR}<30 \mathrm{~dB}$.

In the future, it is expected that a massive number of communication devices and radar systems will need to share the same spectrum. As a result, techniques and approaches to mitigate and minimize interference are of vital importance. It is safe to assume that the new norm will be to have these networks providing different and complementary services, sharing the same bandwidth in an uncoordinated way [96].

\section{Discussion AND Future PERSPECTIVES}

Generally, it can be summarized that the detection of human vital signs by radar techniques involves the selection of the radar type, the appropriate algorithms, as well as the right processing platforms. A special focus, was provided to identify where and how FPGA was implemented in these radars, either as a processing/preprocessing platform, or as the control, or an interfacing device in Section $\mathrm{V}$. The flexibility and reconfigurability nature of the FPGA place it as an excellent candidate for parallel processing and for implementation of algorithms that are computationally more complex.

It can be observed that there is more research directed towards real-time detection, as such feature is highly practical. Real-time detection requires a very powerful processing platform, which is not always affordable for everyday use. Alternative solutions that are being investigated include innovative parallel processing structures on reconfigurable processing devices. Nonetheless, recent developments in reconfigurable devices such as FPGA, enable the processing of multiple operations on hundreds of thousands of logic elements. This opens up opportunities of inventing novel solutions which are capable of processing very complex algorithms to meet the real-time detection requirements.

On the other hand, researchers are still investigating new algorithms to maximize the detection accuracy, at the expense of more complex algorithms. At the same time, more detection scenarios are being experimentally assessed to validate these algorithms in practice. It is demonstrated throughout the survey that the main future trends in vital sign detection using radars is geared towards enabling more practical methods for real-time detection. More practical (and yet challenging) scenarios includes situations where vital signs need to be detected from a person located in a crowded room with multiple people, which is indirectly related to multipath detection. The introduced unwanted signals due to random movements affects detection accuracy due to the additional noise and interference. Other issues include dc offset, coupling issues, position sensitivity, amplitude and phase imbalance and circuits' linearity. To solve these challenges, researchers have introduced more sophisticated algorithms and complex approaches which are computationally-intensive [104], [105], [106], [107]. Such computational requirements can naturally be solved using parallel processing platforms. Other challenges related to circuit design, power consumption etc. have also been addressed by different researchers at different levels [60], [16], [108]. With the recent advances in CMOS processes and technologies, the new challenge for these sophisticated miniaturized designs will then be in terms of low power consumption, which is becoming increasingly stringent. Moreover, the integration of different systems in one platform and interference between different systems are another set of future challenges requiring future research focus. More recently, artificial intelligence in the form of machine learning methods have been introduced to enhance detection accuracy. This brings great potential to the research activities in this field and may enable early warning of fatal situations such as a heart attack or asthma attack [6].

SFCW radar is one of the radar types which have gained the most preference in recent years in the application of vital signs monitoring and detection. The different state-of-the-art literature where SFCW radar was used for human vital sign detection and monitoring, is summarized in Table 9. The articles listed in Table 9 proposed and implemented several algorithms to SFCW, such as CFAR, morphological filtering, numerical method, Fourier transform, singular value decomposition SVD, method of moments, fast multipole method, state-space method, and compressive sensing algorithms. The authors in [55] validated the effectiveness of the proposed algorithms to be used in a MIMO SFCW radar. Through-wall detection of up to three adjacent human subjects can be detected and discriminated based on the reported results. On the other hand, the detection accuracies in [58] were reported to be of $-0.8 \%$ error for RR detection and $0.4 \%$ for $\mathrm{HR}$, with a maximum error of $2 \%$ when the subject is facing away from the radar. Meanwhile in [57], errors of HR detection for multiple targets at different distances from the radar are reported to be about $2 \%$ and $5 \%$ error for the closest and furthest target, respectively. Finally in [2], when a CS algorithm is processed, an error of up to $6.63 \%$ was reported at $80 \%$ of frequency points. The implementation of SSM algorithm, on the contrary, resulted in about $1.2 \%$ error at normal incidence, whereas changing target orientation at different angles produced errors ranging from $0.4 \%$ to $5.7 \%$.

It is important to note that several literature on SFCW radars ([55], [58], [9] and [57]), listed in Table 9, did not specify their processing platforms. In [56] and [2], a standard desktop computer was used as the main processing unit. These articles are focused proving the viability of the algorithm when applied to the SFCW radar for detection and monitoring of HR and RR. Therefore, accuracy is the most important parameter to be reported. Another important observation is that none of these algorithms are designed and implemented on FPGAs. When an 
algorithm is implemented in software loaded into the CPU of a desktop for execution, it implies that the implementation of the algorithm is performed in a sequential manner. In such cases, execution speed, processing time, and the potential to apply such algorithm in real time applications are in doubt. Moreover, the aspects of computational speed, hardware complexity, and cost are elements of less importance in the cited studies. An important final step for such investigations must be tailored towards successfully incorporating these elements (speed, time, hardware, real time) in the performance metrics without deteriorating the state-of-the-art results of reported accuracies.

The discussion of the available algorithms applied for SFCW radars indicated that all of them have been validated to be detecting human vital signs with satisfactory accuracy. However, since these algorithms have neither been implemented on FPGA nor been investigated in terms of speed and processing time, future researches can be directed towards this aspect. Specifically, these algorithms are as follows:

- Constant false alarm rate (CFAR)

- Singular value decomposition (SVD)

- State space method

- Method of moment (MoM)

- $\quad$ Fast multipole method (FMPM)

Investigation on the most efficient approach of implementing these algorithms on FPGA can be further conducted. The aspect of processing speed in using SFCW radar detection for human vital signs can be improved as real time detection with more computationally complex and time-consuming processing should be feasible.

Table 9 Summary of SFCW radar and algorithms used in literature

\begin{tabular}{|c|c|c|c|}
\hline Ref. & Algorithms & Functions/ Advantages & $\begin{array}{l}\text { Frequency } \\
\text { Spectrum }\end{array}$ \\
\hline$[2]$ & 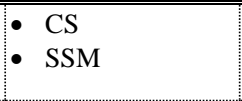 & $\begin{array}{l}\text { To reduce data acquisition time } \\
\text { and to avoid producing inter } \\
\text { modulation products in FFT }\end{array}$ & $2-4 G H z$ \\
\hline [9] & $\begin{array}{l}\text { - FT and hybrid } \\
\text { numerical method } \\
\text { - MoM }\end{array}$ & $\begin{array}{l}\text { To decompose inhomogeneous } \\
\text { object into sub-homogenous } \\
\text { domains and detect the signal in }\end{array}$ & Not reported \\
\hline [55] & • CFAR & $\begin{array}{l}\text { To suppress clutter and mutual } \\
\text { coupling from multiple targets and } \\
\text { to improve weak signals. }\end{array}$ & $40-4400 \mathrm{MHz}$ \\
\hline [56] & SVD and MA & $\begin{array}{l}\text { Clutter reduction techniques to } \\
\text { improve detection accuracy. }\end{array}$ & $1-3 \mathrm{GHz}$ \\
\hline [57] & $\begin{array}{l}\text { - MoM parallel } \\
\text { with FMPM } \\
\text { - SSM }\end{array}$ & $\begin{array}{l}\text { To accelerate computation time } \\
\text { and extract the rate. }\end{array}$ & $2-3 G H z$ \\
\hline [58] & $\bullet \mathrm{CS}$ & $\begin{array}{l}\text { To avoid long data acquisition } \\
\text { time. }\end{array}$ & $2-4 \mathrm{GHz}$ \\
\hline
\end{tabular}

Table abbreviations:

MFC: Morphological Filtering and Clustering

SVD: Singular Value Decomposition

MA: Moving Average

MoM: Method of Moment

FMPM: Fast Multipole Method

SSM: State Space Method

\section{CONCLUSION}

This survey reviewed the recent developments of radars for vital sign detection, with a special focus on the signalprocessing platform and the algorithms' implementation using FPGAs. This review first introduced the various types of radars, architectures, hardware implementation, and methods of detection/sensing. Recent developments suggest that research in this area has prioritized efforts in designing suitable algorithms and processing architectures to meet the challenging real-time detection requirement. These recent trends also suggested that more research is being channeled towards investigating the more complex types of radars (i.e., FMCW and SFCW). Researchers are also striving to acquire better measurement accuracy, while investigating more practical usecases, such as improving the detection distance between radar and target, introducing intentional unwanted movements in the measurements by setting up the experiment in a noisy environment, etc. As research in this area progresses, more attention must be given to implementing real time processing on these (near) practical scenarios. This can be done by introducing novel methods and algorithms for signal extraction on dedicated and powerful processing devices.

\section{REFERENCES}

[1] E. Yavari, "Distortion Reduction and Signal Estimtaion In Doppler Radar Physiological Monitoring Systems," PhD Dissertation, University of Hawaii at Manoa, 2015.

[2] L. Ren, "Noncontact Vital Signs Detection," University of Tennessee USA, 2017.

[3] H.-C. Kuo et al., "A Fully Integrated 60-GHz CMOS DirectConversion Doppler Radar RF Sensor with Clutter Canceller for Single-Antenna Noncontact Human Vital-Signs Detection," IEEE Trans. Microw. Theory Techn., vol. 64, no. 4, pp. 1018-1028, 2016.

[4] N. Andersen et al., "A 118-mW Pulse-Based Radar SoC in 55-nm CMOS for Non-Contact Human Vital Signs Detection," IEEE J. Solid-State Circuits, vol. 52, no. 12, pp. 3421-3433, 2017.

[5] A. D. Droitcour, O. Boric-Lubecke, V. M. Lubecke, J. Lin, and G. T. A. Kovacs, "Range correlation and I/Q performance benefits in single-chip silicon Doppler radars for noncontact cardiopulmonary monitoring," IEEE Trans. Microw. Theory Techn., vol. 52, no. 3, pp. 838-848, 2004.

[6] N. T. P. Van, L. Tang, V. Demir, S. F. Hasan, N. D. Minh, and S. Mukhopadhyay, "Review-microwave radar sensing systems for search and rescue purposes," Sensors, vol. 19, no. 13, pp. 1-24, 2019.

[7] M. Alizadeh, G. Shaker, J. C. M. D. Almeida, P. P. Morita, and S. Safavi-Naeini, "Remote monitoring of human vital signs using mmWave FMCW Radar," IEEE Access, vol. 7, pp. 54958-54968, 2019. T. D. Randeny, "Multi Dimensional Digital Signal Processing In Radar Signature Extraction," PhD Dissertation, University of Akron, Ohaio, 2016.

[9] Q. Nguyen, T. Phan, and O. Kilic, "A hybrid approach and fourier analysis for detection human respiratory rate and heart beat" EEE/ACES International Conference on Wireless Information Technology, ICWITS, 2016.

[10] K. K. Shyu, L. J. Chiu, P. L. Lee, T. H. Tung, and S. H. Yang, "Detection of Breathing and Heart Rates in UWB Radar Sensor Data Using FVPIEF-Based Two-Layer EEMD," IEEE Sens. J., vol. 19, no. 2, pp. 774-784, 2019.

[11] R. J. Fontana, "Recent system applications of short-pulse ultrawideband (UWB) technology," IEEE Trans. Microw. Theory Techn., vol. 52, no. 9 I, pp. 2087-2104, 2004.

[12] B. Li, Z. Zhou, W. Zou, D. Li, and C. Zhao, "Optimal waveforms design for ultra-wideband impulse radio sensors," Sensors, vol. 10, no. 12, pp. 11038-11063, 2010.

[13] J. Chóliz, Á. Hernández, and A. Valdovinos, "A framework for UWB-based communication and location tracking systems for wireless sensor networks," Sensors, vol. 11, no. 9, pp. 9045-9068, 
2011.

[14] Decawave, "UWB Regulations A summary of Worldwid Telecommunications Regulations governing the use of UltraWideband radio." 2018.

[15] European Telecommunications Standards Institute, "Worldwide UWB regulations between 3,1 and 10,6 GHz," vol. 1, pp. 1-64, 2016.

[16] Y. Liu, Y.-H. Liu, S. Sheelavant, M. Mercuri, P. Mateman, and M. Babaie, "An Ultralow Power Burst-Chirp UWB Radar Transceiver for Indoor Vital Signs and Occupancy Sensing in 40-nm CMOS" IEEE Solid-State Circuits Lett., vol. 2, no. 11, pp. 256-259, 2019.

[17] Y. Wang et al., "A 260-mW Ku-Band FMCW Transceiver for Synthetic Aperture Radar Sensor with 1.48-GHz Bandwidth in 65nm CMOS Technology," IEEE Trans. Microw. Theory Techn., vol. 65, no. 11, pp. 4385-4399, 2017.

[18] T. S. Chu, J. Roderick, S. H. Chang, T. Mercer, C. Du, and H. Hashemi, "A short-range UWB impulse-radio CMOS sensor for human feature detection," Dig. Tech. Pap. - IEEE Int. Solid-State Circuits Conf., no. February 2009, pp. 294-295, 2011.

[19] L. Lou et al., "A 253mW/Channel 4TX/4RX Pulsed Chirping Phased- Array Radar TRX in 65nm CMOS for X-Band SyntheticAperture Radar Imaging," IEEE Int. Solid-State Circuits Conf., pp. 160-162, 2018.

[20] D. Obeid et al., "Position-Free Vital Sign Monitoring : Measurements and Processing." HAL, France, p. 25, 2016.

[21] Y. Wang, Q. Liu, and A. E. Fathy, "CW and pulse-Doppler radar processing based on FPGA for human sensing applications," IEEE Trans. Geosci. Remote Sens., vol. 51, no. 5, pp. 3097-3107, 2013.

[22] J. J. Saluja, J. J. Casanova, and J. Lin, "A Supervised Machine Learning Algorithm for Heart-rate Detection Using Doppler MotionSensing Radar," IEEE J. Electromagn. RF Microwaves Med. Biol., vol. 7249, no. c, 2019.

[23] X. Yang, G. Sun, and K. Ishibashi, "Non-contact acquisition of respiration and heart rates using Doppler radar with time domain peak-detection algorithm," in Annual International Conference of the IEEE Engineering in Medicine and Biology Society, EMBS, 2017, pp. 2847-2850.

[24] Meiyu Li and and Jenshan Lin, "Wavelet-Transform-Based DataLength-Variation Technique for Fast Heart Rate Detection Using 5.8GHz CW Doppler Radar," IEEE Trans. Microw. Theory Techn., vol. 2018-Janua, 2018.

[25] A. Vorobyov, E. Daskalaki, and J. Farserotu, "Feasibility of Remote Vital Signs Sensing with a mm-Wave CW Reflectometer," IEEE 38th Int. Conf. Electron. Nanotechnol., pp. 417-421, 2018.

[26] V. L. Petrovic, M. M. Jankovic, A. V. Lupsic, V. R. Mihajlovic, and J. S. Popovic-Bozovic, "High-Accuracy Real-Time Monitoring of Heart Rate Variability Using $24 \mathrm{GHz}$ Continuous-Wave Doppler Radar," IEEE Access, vol. 7, pp. 74721-74733, 2019.

[27] T. W. Hsu and C. H. Tseng, "Compact 24-GHz Doppler radar module for non-contact human vital-sign detection," ISAP 2016 - Int. Symp. Antennas Propag., pp. 994-995, 2017.

[28] M. Nosrati and N. Tavassolian, "High-Accuracy Heart Rate Variability Monitoring Using Doppler Radar Based on Gaussian Pulse Train Modeling and FTPR Algorithm," IEEE Trans. Microw. Theory Techn., vol. 66, no. 1, pp. 556-567, 2018.

[29] P. Li and N. Huo, "A Portable 24GHz Doppler Radar System for Distant Human Vital Sign Monitoring," 5th Int. Conf. Inf. Sci. Control Eng., pp. 1050-1052, 2019.

[30] C. Li et al., "A method for remotely sensing vital signs of human subjects outdoors," Sensors, vol. 15, no. 7, pp. 14830-14844, 2015.

[31] F. Quaiyum, N. Tran, T. Phan, P. Theilmann, A. E. Fathy, and O. Kilic, "Electromagnetic Modeling of Vital Sign Detection and Human Motion Sensing Validated by Noncontact Radar Measurements," IEEE J. Electromagn. RF Microwaves Med. Biol., vol. 2, no. 1, pp. 40-47, 2018.

[32] L. Qiu, T. Jin, J. Zhang, B. Lu, and Z. Zhou, "A singular spectrum analysis based human life signal detection," Progress In Electromagnetics Research Symposium, 2016, pp. 4295-4298.

[33] M. Mercuri et al., "A Direct Phase-Tracking Doppler Radar Using Wavelet Independent Component Analysis for Non-Contact Respiratory and Heart Rate Monitoring," IEEE Trans. Biomed. Circuits Syst., vol. 12, no. 3, pp. 632-643, 2018.

[34] T. Zhangt, G. Valerio, J. Sarrazin, and D. Istrate, "Non-contact estimation at $60 \mathrm{GHz}$ for human vital signs monitoring using a robust optimization algorithm," IEEE Antennas Propag. Soc. Int. Symp., pp. 1165-1166, 2016.
[35] A. Rahman, "Doppler Radar Techniques for Distinct Respiratory Pattern Recognition and Subject Identification," PhD Dissertation, The University of Hawai' $i, 2017$.

[36] R. Qian, T. Jin, H. Li, and Y. Dai, "WT-Based Data-Length-Variation Technique for Fast Heart Rate Detection," in Progress in Electromagnetics Research Symposium, 2018, vol. 2018-Augus, pp. 399-404.

[37] Y. Rong and D. W. Bliss, "Harmonics-based multiple heartbeat detection at equal distance using UWB impulse radar," IEEE Radar Conf., no. 1, pp. 1101-1105, 2018.

[38] F. Khan and S. H. Cho, "A detailed algorithm for vital sign monitoring of a stationary/non-stationary human through IR-UWB radar," Sensors, vol. 17, no. 2, 2017.

[39] C. Eren, S. Karamzadeh, and M. Karatal, "The artifacts of human physical motions on vital signs monitoring," in Scientific Meeting on Electrical-Electronics and Biomedical Engineering and Computer Science, 2019, no. 1.

[40] M. L. Tudose, A. Anghel, R. Cacoveanu, and M. Datcu, "Pulse Radar with Field-Programmable Gate Array Range Compression for Real Time Displacement and Vibration Monitoring," Sensors, vol. 19, no. $1,2018$.

[41] H.-S. Cho and Y.-J. Park, "Novel design for heart rate detection using UWB impulse radar on Android platform," IEEJ Trans. Electr. Electron. Eng., vol. 13, no. 5, pp. 799-800, 2018.

[42] E. Schires, P. Georgiou, and T. S. Lande, "Vital sign monitoring through the back using an UWB impulse radar with body coupled antennas," IEEE Trans. Biomed. Circuits Syst., vol. 12, no. 2, pp. 292-302, 2018.

[43] M. A. Aseeri, "Feasibility of mapping a pulsed RADAR system to FPGA-ASIC and hybrid architectures," IEEE Int. Conf. Emerg. Technol. Innov. Bus. Pract. Transform. Soc., no. September, pp. 8589, 2016.

[44] K.-C. Tsao, L. Lee, T.-S. Chu, and Y.-H. Huang, "A two-stage reconstruction processor for human detection in compressive sensing CMOS radar," Sensors, vol. 18, no. 4, 2018.

[45] C.-H. Hsieh, Y.-F. Chiu, Y.-H. Shen, T.-S. Chu, and Y.-H. Huang, "A UWB Radar Signal Processing Platform for Real-Time Human Respiratory Feature Extraction Based on Four-Segment Linear Waveform Model," IEEE Trans. Biomed. Circuits Syst., vol. 10, no. 1, pp. 219-230, 2016.

[46] Y. Rong and D. W. Bliss, "Direct RF Signal Processing for HeartRate Monitoring Using UWB Impulse Radar," in Conference on Signals, Systems and Computers, 2019, vol. 2018-Octob, pp. 12151219.

[47] H.-S. Cho, Y.-J. Park, and H.-K. Lyu, "Robust heart rate detection method using UWB impulse radar," in International Conference on Information and Communication Technology Convergence, 2016, pp. $1138-1142$.

[48] N. Shruthi, P. Mathur, and D. G. Kurup, "Performance of Ultra Wideband (UWB) pulsed Doppler Radar for heart rate and respiration rate monitoring in Noise," in International Conference on Advances in Computing, Communications and Informatics, 2018, pp. 722-725.

[49] L. Ren, H. Wang, K. Naishadham, O. Kilic, and A. E. Fathy, "PhaseBased Methods for Heart Rate Detection Using UWB Impulse Doppler Radar," IEEE Trans. Microw. Theory Techn., vol. 64, no. 10, pp. 3319-3331, 2016.

[50] L. Ren, Y. S. Koo, H. Wang, Y. Wang, Q. Liu, and A. E. Fathy, "Noncontact multiple heartbeats detection and subject localization using UWB impulse Doppler radar," IEEE Microw. Wirel. Components Lett., vol. 25, no. 10, pp. 690-692, 2015.

[51] R. Ernst, "Vital Sign Radar," Master Thesis, Halmstad University, Sweden, 2016.

[52] R. Qian, D. Jiang, and W. Fu, "FPGA implementation of closed-loop compensation for LFMCW signal non-linear distortions," IET Signal Process., vol. 13, no. 2, pp. 192-198, 2019.

[53] T. M. Samy, M. S. Abdel-Latif, S. A. Elgamel, and F. M. Ahmed, "FPGA implementation of pulsed noise interference against LFM radar," in 12th International Conference on Computer Engineering and Systems, 2018, vol. 2018-Janua, pp. 695-700.

[54] H. Eugin and J. Lee, "Hardware architecture design and implementation for FMCW radar signal processing algorithm," Conf. Des. Archit. Signal Image Process. DASIP, vol. 2015-May, pp. 1-6, 2015.

[55] F. Liang et al., "Detection of Multiple Stationary Humans Using UWB MIMO Radar," Sensors, vol. 16, no. 11, 2016. 
[56] A. N. Gaikwad and K. S. Dongre, "Improvement in detection of human life sign signal hidden behind the wall using clutter reduction technique," in International Conference on Emerging Trends in Communication Technologies, 2017.

[57] T. Phan, O. Kilic, S. Nahar, L. Ren, and A. E. Fathy, "Accuracy Investigation of SFCW Radar in Human Vital Signs Detection for Subject's Relative Position," in IEEE Antennas and Propagation Society International Symposium, Proceedings, 2017, vol. 2017Janua, pp. 577-578.

[58] A. E. Fathy, L. Ren, S. Nahar, and O. Kilic, "Overview of human vital signs detection using radar techniques," in IEEE Antennas and Propagation Society International Symposium, Proceedings, 2017, vol. 2017-Janua, pp. 1229-1230.

[59] J. H. Park, Y. J. Jeong, G. E. Lee, J. T. Oh, and J. R. Yang, "915$\mathrm{MHz}$ continuous-wave Doppler radar sensor for detection of vital signs," Electronics, vol. 8, no. 8, 2019.

[60] Y. Liu et al., "A $680 \mu \mathrm{W}$ Burst-Chirp UWB Radar Transceiver for Vital Signs and Occupancy Sensing up to $15 \mathrm{~m}$ Distance," IEEE Int. Solid-State Circuits Conf. -, pp. 166-168, 2019.

[61] M. Mercuri, Y. Liu, S. Sheelavant, S. Polito, T. Torfs, and C. Van Hoof, "Digital Linear Discrete FMCW Radar for Healthcare Applications," pp. 144-147, 2019.

[62] B. Ria Ghosh, "Hardware Implementation of Real Time Beat Detection and Classification Algorithm for automated ECG Analysis," Master Thesis, The University of Texas at Dallas, USA, 2018.

[63] A. N. Gaikwad and K. S. Dongre, "Improvement in detection of human life sign signal hidden behind the wall using clutter reduction technique," in International Conference on Emerging Trends in Communication Technologies, ETCT 2016, 2017.

[64] M. Mercuri, I. R. Lorato, Y. H. Liu, F. Wieringa, C. Van Hoof, and T. Torfs, "Vital-sign monitoring and spatial tracking of multiple people using a contactless radar-based sensor," Nat. Electron., vol. 2, no. 6, pp. 252-262, 2019.

[65] J. Tu, T. Hwang, and J. Lin, "Respiration Rate Measurement under 1-D Body Motion Using Single Continuous-Wave Doppler Radar Vital Sign Detection System," IEEE Trans. Microw. Theory Techn., vol. 64, no. 6, pp. 1937-1946, 2016.

[66] C. Li and J. Lin, "Random body movement cancellation in Doppler radar vital sign detection," IEEE Trans. Microw. Theory Techn., vol. 56, no. 12, pp. 3143-3152, 2008.

[67] C. Gu, G. Wang, Y. Li, T. Inoue, and C. Li, "A hybrid radar-camera sensing system with phase compensation for random body movement cancellation in doppler vital sign detection," IEEE Trans. Microw. Theory Techn., vol. 61, no. 12, pp. 4678-4688, 2013.

[68] Y. Quan, Y. Li, X. Gao, and M. Xing, "FPGA Implementation of Real-Time Compressive Sensing with Partial Fourier Dictionary," Int. J. Antennas Propag., vol. 2016, 2016.

[69] A. Jarrah and M. M. Jamali, "Reconfigurable FPGA/GPU-based architecture of block compressive sampling matching pursuit algorithm," J. Circuits, Syst. Comput., vol. 24, no. 4, pp. 1-23, 2015.

[70] Q. Liang et al., "Research on non-contact monitoring system for human physiological signal and body movement," Biosensors, vol. 9, no. 2, 2019.

[71] B. Erol, "Radar-based Human Activity Recognition Algorithms using Machine Learning and Deep Neural Networks," Master Thesis, Villanova University,Pennsylvania, 2018.

[72] R. Rasu, P. S. Sundaram, and N. Santhiyakumari, "FPGA based noninvasive heart rate monitoring system for detecting abnormalities in Fetal," Int. Conf. Signal Process. Commun. Eng. Syst., pp. 375-379, 2015.

[73] N. S. Risman, S. N. Yassin, N. A. Nayan, S. C. Wei, and Y. W. Hau, "Implementation of heart rate variability analysis algorithm on FPGA platform," Int. J. Appl. Eng. Res., vol. 11, no. 12, pp. 7809-7814, 2016.

[74] J. Kamble, I. A. Pasha, and M. Madhavilatha, "FPGA implementation of digital modulation technique for HRR target detection," Indian J. Sci. Technol., vol. 8, no. 24, 2015.

[75] H. Suarez and Y. R. Zhang, "System-on-chip architecture and validation for real-time transceiver optimization: APC implementation on FPGA," in Proceedings of SPIE - The International Society for Optical Engineering, 2015, vol. 9461.

[76] C. A. Parmar, B. Ramanadham, and A. D. Darji, "FPGA implementation of hardware efficient adaptive filter robust to impulsive noise," IET Comput. Digit. Tech., vol. 11, no. 3, pp. 107-
116, 2017.

[77] S. Srinivasa Rao and P. Siddaiah, "Design and implementation of four-phase sequences on FPGA using modified particle swarm optimization for radar applications," Int. J. Appl. Eng. Res., vol. 12, no. 11, pp. 2907-2915, 2017.

[78] J. Zhang, R. Zhang, and Y. Dai, "Design and FPGA implementation of DDS based on waveform compression and Taylor series," in 29th Chinese Control and Decision Conference, CCDC 2017, 2017, pp. 1301-1306.

[79] W. Andre and O. Couillard, "Design and implementation of a new architecture of a real-time reconfigurable digital modulator on FPGA," Electron. Lett., vol. 54, no. 18, pp. 1094-1095, 2018.

[80] V. Thakur, A. K. Verma, P. Jena, and G. S. Prasad, "Design and implementation of FPGA based Digital Pulse Compression via fast convolution using FFT-OS method," Int. Conf. Microwave, Opt. Commun. Eng., pp. 455-458, 2016.

[81] N. Pallavi, P. Anjaneyulu, P. B. Reddy, V. Mahendra, and R. Karthik, "Design and implementation of linear frequency modulated waveform using DDS and FPGA," in International Conference on Electronics, Communication and Aerospace Technology, 2017, vol. 2017-Janua, pp. 237-241.

[82] M. R. Bales and S. C. Sutphin, "FPGA architecture for real-time wideband waveform synthesis," in IEEE National Radar Conference - Proceedings, 2015, vol. 2015-June, no. June, pp. 605-610.

[83] N. ul Azim and W. Jun, "FPGA based hardware optimized implementation of signal processing system for LFM pulsed radar," Infrared, Millimeter-Wave, Terahertz Technol. IV, vol. 10030, p. 100302U, 2016.

[84] J. Mangala and J. Manikandan, "FPGA implementation of reconfigurable modulation system," in International Conference on Advances in Computing, Communications and Informatics, 2015, pp. 493-500.

[85] P. J. Soh, G. A. E. Vandenbosch, M. Mercuri, and D. M. M. P. Schreurs, "Wearable Wireless Health Monitoring," IEEE Microw. Mag., vol. 16, no. 4, pp. 55-70, 2015.

[86] C. Chen et al., "TR-BREATH: Time-Reversal Breathing Rate Estimation and Detection," IEEE Trans. Biomed. Eng., vol. 65, no. 3, pp. 489-501, 2018.

[87] C. Li et al., "Overview of Recent Development on Wireless Sensing Circuits and Systems for Healthcare and Biomedical Applications," IEEE J. Emerg. Sel. Top. Circuits Syst., vol. 8, no. 2, pp. 165-177, 2018.

[88] J. Liu, Y. Wang, Y. Chen, J. Yang, X. Chen, and J. Cheng, "Tracking vital signs during sleep leveraging off-the-shelf WiFi," Proc. Int. Symp. Mob. Ad Hoc Netw. Comput., vol. 2015-June, pp. 267-276, 2015.

[89] H. Abdelnasser, K. A. Harras, and M. Youssef, "UbiBreathe: A ubiquitous non-invasive wifi-based breathing estimator," Proc. Int. Symp. Mob. Ad Hoc Netw. Comput., vol. 2015-June, pp. 277-286, 2015.

[90] V. Lubecke, O. Boric-Lubecke, and E. Beck, "A compact low-cost add-on module for Doppler radar sensing of vital signs using a wireless communications terminal," IEEE MTT-S Int. Microw. Symp. Dig., vol. 3, pp. 1767-1770, 2002.

[91] C. G. Bilich, "Bio-medical sensing using Ultra Wideband communications and radar technology: A feasibility study," 2006 Pervasive Heal. Conf. Work., 2006.

[92] B. Gupta, D. Valente, E. Cianca, and R. Prasad, "FM-UWB for radar and communications in medical applications," 1st Int. Symp. Appl. Sci. Biomed. Commun. Technol., 2008.

[93] M. Mercuri, P. Karsmakers, B. Vanrumste, P. Leroux, and D. Schreurs, "Biomedical wireless radar sensor network for indoor emergency situations detection and vital signs monitoring," IEEE Top. Conf. Biomed. Wirel. Technol. Networks, Sens. Syst., pp. 32-35, 2016.

[94] M. Mercuri et al., "Analysis of an indoor biomedical radar-based system for health monitoring," IEEE Trans. Microw. Theory Techn., vol. 61, no. 5, pp. 2061-2068, 2013.

[95] C. Li, J. Cummings, J. Lam, E. Graves, and W. Wu, "Radar remote monitoring of vital signs," IEEE Microwave Magazine, vol. 10, no. 1, pp. 47-56, 2009.

[96] A. Munari, N. Grosheva, L. Simić, and P. Mähönen, "Performance of Radar and Communication Networks Coexisting in Shared Spectrum Bands," IEEE Int. Symp. Pers. Indoor Mob. Radio Commun. PIMRC, pp. 1-8, 2019. 
[97] D. Garmatyuk and J. Schuerger, "Conceptual Design of A Dual Use Radar/Communication System Based on OFDM," in IEEE Military Communications Conference, 2008.

[98] Y. Zhu, Y. Zhu, B. Y. Zhao, and H. Zheng, "Reusing 60GHz radios for mobile radar imaging," Proc. Anпи. Int. Conf. Mob. Comput. Networking, MOBICOM, vol. 2015-Septe, pp. 103-116, 2015.

[99] M. Mercuri et al., "Dual-mode wireless sensor network for real-time contactless in-door health monitoring," 2015 IEEE MTT-S Int. Microw. Symp. IMS 2015, pp. 8-11, 2015.

[100] P. Kumari, J. Choi, N. Gonzalez-Prelcic, and R. W. Heath, "IEEE 802.11ad-Based Radar: An Approach to Joint Vehicular Communication-Radar System," IEEE Trans. Veh. Technol., vol. 67, no. 4, pp. 3012-3027, 2018.

[101] F. Guidi, A. Guerra, and D. Dardari, "Personal mobile radars with millimeter-wave massive arrays for indoor mapping," IEEE Trans. Mob. Comput., vol. 15, no. 6, pp. 1471-1484, 2016.

[102] A. Khawar, A. Abdel-Hadi, and T. C. Clancy, "Spectrum sharing between S-band radar and LTE cellular system: A spatial approach," IEEE Int. Symp. Dyn. Spectr. Access Networks, DYSPAN 2014, pp. 7-14, 2014.

[103] M. Mehrnoush and S. Roy, "Coexistence of WLAN Network with Radar: Detection and Interference Mitigation," IEEE Trans. Cogn. Commun. Netw., vol. 3, no. 4, pp. 655-667, 2017.

[104] N. M. Thamrin, M. A. Haron, and F. A. Ruslan, "A field programmable gate array implementation for biomedical System-onChip (SoC)," in Proceedings - 2011 IEEE 7th International Colloquium on Signal Processing and Its Applications, CSPA 2011, 2011.

[105] W. Hu, C. C. Lin, L. Y. Shyu, C. Yuan, and C. Li, "An Implementation of a Real-Time and Parallel Processing ECG Features Extraction Algorithm in a Field Programmable Gate Array (FPGA)," in Computing in Cardiology, 2011, pp. 38-41.

[106] K. Tuppurainen et al., "Respiration rate monitoring methods: a review." HAL, UK, p. 48, 2011.

[107] B. S. Jensen, T. K. Johansen, and V. Krozer, "Microwave Instrument for Human Vital Signs Detection and Monitoring," Ph.D. dissertation, Technical University of Denmark, 2012.

[108] K. Lakhotia, G. Caffarena, A. Gil, D. G. Marquez, A. Otero, and M. P. Desai, "Low-Power, Low-Latency Hermite Polynomial Characterization of Heartbeats Using a Field-Programmable Gate Array," 4th International Conference on Bioinformatics and Biomedical Engineering, 2016, vol. 9656, pp. 266-276.

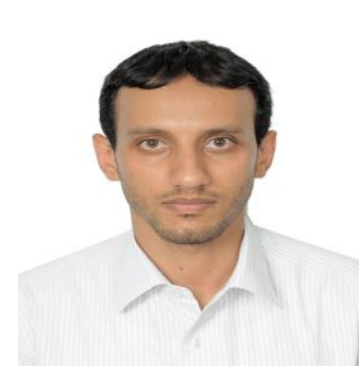

Ameen Bin Obadi is a PhD student at University of Perlis, Malaysia (UNIMAP). Ameen obtained his BSc. Degree (Hons) at King Fahd University of Petroleum and Minerals (KFUPM), Saudi Arabia (2010) in electrical engineering and his MSc. Degree as well at KFUPM, Saudi Arabia (2014) in electrical engineering (Analog \& digital

electronics). He worked as an IP Commercialization Executive at Dhahran Techno-Valley Company and as a manager of technology venture development. His experience in this area ranges across managing a portfolio of technologies including evaluation, marketing, technology advancements, licensing and/or spin-outformation. He backed up his experience in technology commercialization with training sessions in Europe and the US provided by ASTP-PROTON and AUTM. He started his career at the Japanese company JGC Gulf in the oil \& gas EPC industry as an electrical engineer in Khobar. He then worked at Schneider Electric in the energy division handling Saudi Electricity Company contractors' portfolio in the main office in Riyadh.

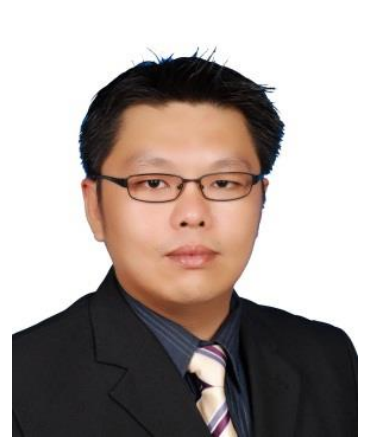

Ping Jack Soh, PhD, C. Eng (S '10, M'14, SM '15) was born in Sabah, Malaysia. He received the B. Eng and M. Eng degrees in Electrical Engineering (Telecommunication) from Universiti Teknologi Malaysia (UTM) in 2002 and 2006, respectively, and the $\mathrm{PhD}$ degree in Electrical Engineering from $\mathrm{KU}$ Leuven, Belgium in 2013.

He is currently the Head of the Advanced Communication Engineering (ACE) Research Centre, Universiti Malaysia Perlis (UniMAP), an Associate Professor at the School of Computer and Communication Engineering (SCCE), UniMAP, and also a Research Affiliate at KU Leuven, Belgium. Prior to that, he was a Test Engineer in Venture Corp. from 2002 to 2004 and was an R\&D Engineer in Motorola Solutions Malaysia in 2005. From 2006, he joined SCCE-UniMAP as a Lecturer, and also served as the Deputy Director of the Centre for Industrial Collaboration (CIC) between 2007 and 2009. He went on leave from UniMAP in 2009 to pursue his Ph.D. and research attachment in KU Leuven, Belgium. He was first a Research Assistant (20092013), then a Postdoctoral Research Fellow (2013-2014) and is currently a Research Affiliate in the ESAT-TELEMIC Research Division. Upon his return to UniMAP in 2014, he resumed his role as a Senior Lecturer, and concurrently served as the Deputy Dean of the university's Research Management and Innovation Center (RMIC) between 2014 and 2017. He researches actively in his areas of interest: wearable and flexible antennas/metasurfaces; on-body communication; electromagnetic safety and absorption; and wireless and radar techniques for healthcare applications. He is also an Associate Editor of the International Journal of Numerical Modelling: Electronic Networks, Devices and Fields (Wiley). His involvement in several industrial research projects have resulted in a granted patent and five other filed patents.

Dr. Soh was the recipient of the IEEE Antennas and Propagation Society (AP-S) Doctoral Research Award in 2012, the IEEE Microwave Theory and Techniques Society (MTT-S) Graduate Fellowship for Medical Applications in 2013 and the International Union of Radio Science (URSI) Young Scientist Award in 2015. He was also the Second Place Winner of the IEEE Presidents' Change the World Competition in 2013. Two of his (co)authored journals were awarded the IEEE AP/MTT/EMC Malaysia Joint Chapter's Best Paper Award in 2018 and 2019, and another two journals were also awarded the CST University Publication Award in 2012 and 2011. As project advisor, his supervised students have also won prizes such as the IEEE MTT-S Undergraduate Scholarship in 2019 and 2016, First Place in the Innovate Malaysia Design Competition (Motorola Track) in 2016 and the First Place in the IEEE Malaysia Section Final Year Project Competition (Telecommunication Track) in 2015. He is a Chartered Engineer registered with the UK Engineering Council; a Professional Technologist registered with the Malaysia Board of Technologist (MBOT); a Senior Member of the IEEE, a Member of the IET and the URSI. He also serves in the IEEE 
MTT-S Education Committee, and the IEEE MTT-S Meetings and Symposia $(\mathrm{M} \& \mathrm{~S})$ Committee.

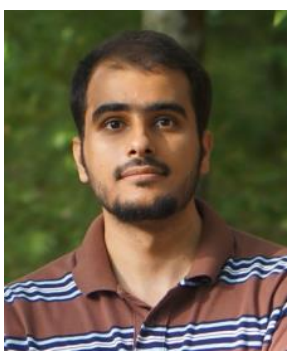

Dr. Omar Aldayel is an Assistant Professor in the Department of Electrical Engineering at King Saud University, Riyadh, Saudi Arabia. He received B.S. degree from King Saud University, Riyadh, Saudi Arabia, M.S. degree from KTH-- the Royal Institute of Technology, Stockholm, Sweden and Ph.D. degree in Electrical Engineering from The Pennsylvania State University, University Park, PA, USA in 2007, 2011 and 2017, respectively. He worked at King Saud University as a lecturer, Riyadh, Saudi Arabia, from 2011 to 2013. Dr. Aldayel's research interests include radar signal processing, detection and estimation, and convex optimization.

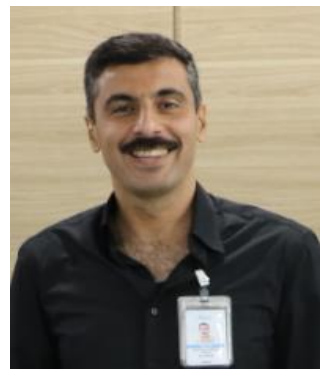

Dr. Muataz H. Salih received the B.Sc. and M.Sc. degrees from the Dept. of Computer Engineering from University of Technology, Iraq, in 1998 and 2002, respectively. In Sept 2013, he earned a PhD degree in Computer Engineering, with specialization in FPGA Embedded Multiprocessor SoC. From Sept. 1998 to March 2003, he was a research engineer in Military Industrialization Corporation of Iraq. From Oct. 2003 to June 2008 , he was a lecturer and manager of engineering faculty's LABS in the faculty of Engineering of Al-Kalamoon private university, Syria. From July 2008 to July April 2011, he was a Researcher at Underwater Robotic Research Group at USM. Nov. 2013 to Oct 2018, he is a Senior lecturer at UniMAP, Malaysia. Since Oct. 2018, he is an FPGA Senior Staff Engineer Manager at Flex company. He is IR4.0 and Intelligent Automation lead of Flex Penang Cluster. He is SMIEEE, CEng, MIET and IACSIT Senior Member. His research interests and working area on designing digital systems using FPGA technology, FPGA-SoC design, embedded systems, computer system architecture, microprocessor architecture, active jamming system for laser missiles, IR4.0 transformation, Cybersecurity and Intelligent Automation.

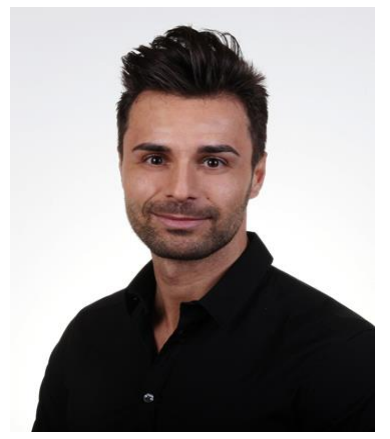

Marco Mercuri was born in Calabria, Italy in 1985. He received the B.S. and M.S. degrees in electronic engineering from the University of Calabria (UNICAL), Italy, in 2006 and 2009, respectively, and the Ph.D. degree in electronic engineering from $\mathrm{KU}$ Leuven, Belgium, in 2015. He is currently Senior Researcher at imec - Netherlands, Eindhoven, Netherlands. His research interests include microwave/RF radar systems and algorithms, contactless health monitoring, biomedical applications, embedded systems and wireless sensor networks. Dr. Mercuri is the author and co-author of over 50 publications in peer-reviewed journals, conferences, and books. He holds several patents on radars for biomedical applications. He was the recipient of the ISSCC 2019 Demonstration Session Certificate of Recognition, 2013 IEEE MTT-S Graduate Fellowship Award, and he obtained the second place both to the 2013 IEEE President's Change the World Competition (PCW) and to the 2013 IEEE MTT-S YouTube/YouKu Video Competition.

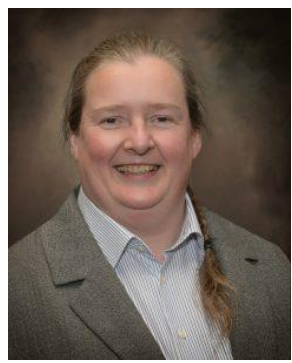

Prof. Dominique M. M.-P. Schreurs (S'90-M'97-SM'02-F'12) received the M.Sc. degree in electronic engineering and the Ph.D. degree from the University of Leuven (KU Leuven), Leuven, Belgium, in 1992 and 1997, respectively. She has been a Visiting Scientist with Agilent Technologies, Santa Rosa, CA, USA, ETH Zurich, Zurich, Switzerland, and the National Institute of Standards and Technology, Boulder, CO, USA. She is currently a Full Professor with KU Leuven, where she is also the Chair of the Leuven LICT. Her current research interests include the microwave and millimeter-wave characterization and modeling of transistors, nonlinear circuits, and bioliquids, and system design for wireless communications and biomedical applications. Prof. Schreurs served as President of the IEEE Microwave Theory and Techniques Society (April 2018-2019), and priory was Editor-in-Chief of the IEEE Transactions on Microwave Theory and Techniques as well as IEEE MTT-S Distinguished Microwave Lecturer. She also served as President of the ARFTG organization (2018-2019) and was General Chair of the 2007, 2012, and 2018 Spring ARFTG Conferences. In 2020, she serves as TPC chair of the European Microwave Conference (EuMC) and is also conference co-chair of the IEEE International Microwave Biomedical Conference (IMBioC). 